\title{
DESENREDANDO LAS IDENTIDADES SOBERANISTAS VASCA Y \\ CATALANA: UN ANÁLISIS DE REDES SOCIALES DE LAS ETIQUETAS DE TWITTER \#BASQUESDECIDE Y \#UP4FREEDOM
}

\section{Unravelling the Basque and Catalan "Soberanist" Identities: a Social Network Analysis of the Twitter Hashtags \#BasquesDecide and \#Up4Freedom}

\author{
Jordi Morales i Gras* \\ *Universidad del País Vasco / Euskal Herriko Unibertsitatea \\ morales.jordi@gmail.com
}

Palabras clave

Análisis de redes

sociales

Twitter

País Vasco

Cataluña

Keywords

Social Network

Analysis

Twitter

Basque Country

Catalonia

\section{$\underline{\text { Resumen }}$}

La intención de este artículo es identificar y comparar mediante técnicas de Análisis de Redes Sociales algunos de los agentes que contribuyen de manera relevante al proceso de configuración de identidades políticas soberanistas en el País Vasco y en Cataluña, así como atender a las relaciones establecidas entre los participantes de los debates bajo las etiquetas \#BasquesDecide y \#Up4Freedom. El artículo muestra que existen diferencias sustanciales entre las comunicaciones establecidas en Twitter bajo ambas etiquetas tanto por lo que respecta a la participación de sus usuarios como por el tipo de usuarios que gozan de mayor influencia o poder en las redes y las dinámicas que configuran sus comunidades.

\section{Abstract}

The goal of this article is to identify and compare through Social Network Analysis' techniques some of the agents that significantly contribute to the formation process of the "soberanist" (i.e. secessionist, separatist) political identities in the Basque Country and in Catalonia. We will analyse as well the relations established among the participants of the debates under the hashtags \#BasquesDecide and \#Up4Freedom. As we will show, there are substantial differences between the communications established in Twitter under both hashtags in terms of user participation, the kind of users that hold greater levels of influence or power within the networks and the dynamics that configure their communities.

Morales i Gras, J., 2015, "Desenredando las identidades soberanistas vasca y catalana: un Análisis de Redes Sociales de las etiquetas de Twitter \#BasquesDecide y \#Up4Freedom", en Papeles del CEIC, vol. 2015/2, no 128, CEIC (Centro de Estudios sobre la Identidad Colectiva), Universidad del País Vasco, http://dx.doi.org/10.1387/pceic.14161 


\section{INTRODUCCIÓN}

El domingo 8 de Junio de 2014 coincidían dos movilizaciones distintas, convocadas desde dos territorios europeos distintos. Sin embargo, ambas guardaban similitudes importantes. Por un lado, el grupo vasco Gure Esku Dago convocaba una cadena humana por el derecho a decidir del pueblo vasco sobre su soberanía política. Según las cifras proporcionadas por la propia organización, unas 150.000 personas acudieron a la convocatoria (Gure Esku Dago, 2014). Simultáneamente, la asociación catalana Òmnium Cultural promovía una jornada castellera internacional. Según la misma organización, más de 5.000 personas levantaron castillos humanos en ocho capitales europeas y muchas otras ciudades con la intención de hacer llegar al mundo la reivindicación de poder someter la soberanía política de Cataluña a votación (Òmnium Cultural, 2014).

Ambas convocatorias tuvieron una incidencia considerable en la red social de microblogging Twitter. Las organizaciones convocantes crearon y fomentaron el uso de las etiquetas \#GureEskuDago y \#BasquesDecide en el caso de la cadena humana, y \#CatalansWantToVote y \#Up4Freedom en el caso de la jornada internacional de castillos humanos. Los algoritmos de promoción de contenidos de Twitter hicieron que las cuatro etiquetas llegaran a ser "tendencia" estatal e incluso mundial durante la jornada. Se trata de cuatro etiquetas con una carga identitaria muy connotada políticamente. Pero en este artículo no se discutirá desde una perspectiva política el estado del arte de las categorías de identificación vasca y catalana, ni mucho menos las razones para apoyar o no los proyectos soberanistas contemporáneos en sendos territorios. Por el contrario, serán abordadas de forma comparativa algunas de las consecuencias de la creciente hegemonía de formas de comunicación cada vez más horizontales en el proceso de formación de identidades políticas soberanistas, cuya demanda de participación es también inaudita.

La intención de este artículo es identificar y comparar, a partir de dos instancias comunicativas, algunos de los agentes que contribuyen de manera relevante al proceso de configuración de identidades políticas soberanistas en el País Vasco y en Cataluña, así como las relaciones establecidas entre los participantes de los debates bajo las etiquetas \#BasquesDecide y \#Up4Freedom. Para ello, se prestará especial 
atención al tipo de agentes que median la comunicación de manera más destacada, ya sean estos usuarios de Twitter relativos a individuos, a organizaciones o a medios de comunicación, puesto que diferencias significativas a este nivel podrían implicar modelos dispares de desarrollo identitario en el medio digital que deberían ser tomados en consideración en una investigación de mayor alcance ${ }^{1}$. Otro factor fundamental a observar es la similitud o disimilitud no solo en términos del tipo de liderazgo o referencialidad que estructura los grupos de nodos, sino el número de agentes que cooperan y compiten simultáneamente por la hegemonía de la red.

La teoría de redes sociales supone una perspectiva simultáneamente relacional y estructural de lo social (Requena Santos, 1989; Lozares, 1996; Molina, 2001; Rodríguez Díaz, 2005). En este sentido, la perspectiva teórica y epistemológica de este artículo que es básicamente empírico tiene que ser situada a caballo entre las expresiones sociológicas que entienden que es la interacción entre entidades sociales -individuales y colectivas - la que da lugar a la unidad de análisis irreductible y las perspectivas psicosociológicas que ponen su énfasis en los procesos de gestación de realidades sociales operativas. Dada la escasez de trabajos empíricos con propósitos análogos al presente y que utilicen información de Twitter para evaluar fenómenos identitarios, se tomará como estrategia la construcción de un marco teórico ad-hoc y su articulación en términos empíricos.

En primer lugar se presenta el paradigma de la auto-comunicación masiva de Manuel Castells, propio de la Sociedad-Red. También se proporciona un marco interpretativo en clave identitario para el análisis psicosociológico de las comunicaciones creadas en redes sociales virtuales recurriendo, para ello, a modelos teóricos diversos como el paradigma de la Identidad Social de Henri Tajfel (1974) y John C. Turner entre otros (1994), la Teoría del Actor-Red de Bruno Latour (2005), o la teoría narrativa sobre identidades colectivas de Klaus Eder (2009). Seguidamente, se presentan los aspectos metodológicos y técnicos necesarios para entender el abordaje empírico de los conjuntos de datos obtenidos y que han sido tratados con herramientas propias del Análisis

\footnotetext{
${ }^{1}$ Esta investigación forma parte de un proyecto de Tesis Doctoral que persigue, mediante un análisis más sistemático y constante en el tiempo, y también mediante distintas perspectivas metodológicas, una comprensión del fenómeno de mayor amplitud y profundidad.
} 
de Redes Sociales. Se discuten los resultados obtenidos y sus implicaciones después de haberlos presentado mostrando que existen diferencias sustanciales entre las comunicaciones establecidas en Twitter bajo las etiquetas \#BasquesDecide y \#Up4Freedom tanto en lo que respecta a la participación de sus usuarios como al tipo de usuarios que gozan de mayor influencia o poder en las redes y las dinámicas que configuran sus comunidades. Finalmente se apuntan algunas conclusiones de la investigación.

\section{ReDES, IDENTIDAD y COMUNIDADES NARRATIVAS}

\subsection{La identidad en la enredada era de la auto-comunicación masiva}

Las sociedades contemporáneas están enredadas. Tal afirmación es hoy un lugar común en la ciencia social, principalmente gracias a la labor del sociólogo Manuel Castells en su caracterización de la "Sociedad-Red" $(2000,2009,2010)$. Con respecto a los fenómenos identitiarios, en la Sociedad-Red el sustancialismo de la primera modernidad se diluye a través de los medios tecnológicos que permiten relaciones indirectas cada vez más fluidas entre individuos (Castells, 2000). Emergen nuevas identidades, pero éstas ya no se parecen tanto a aquellas referidas por los sociólogos modernistas e instrumentalistas que fueran diseñadas por las inteligencias o élites nacionales y difundidas entre una población pendiente de nacionalizar (Anderson, 1991; Gellner, 2006; Hechter y Levi, 1979; Hobsbawm y Ranger, 1992), puesto que se trata de "proyectos de identidad" que sus mismos portadores construyen en base a los materiales de los que disponen y con las que se persigue la transformación de la estructura social en su conjunto (Castells, 2009). Más adelante mostramos cómo el paradigma psicosociológico de la Identidad Social puede contribuir a la caracterización de los procesos y las dinámicas que dan lugar a los proyectos identitarios descritos por Castells.

En la Sociedad-Red las estructuras de poder devienen o se mantienen dominantes sólo si adquieren propiedades tales como centralidad en la red, la capacidad de influir en redes o la habilidad de crearlas y expandirlas. La estrategia clásica de los medios de comunicación tradicionales y verticales de ceñirse a una suerte limitada de perspectivas ideológicamente connotadas se ve reemplazada por una 
estrategia más horizontal y centrífuga, al mismo tiempo que los consumidores de comunicación se vuelven cada vez más individualistas y selectivos (Castells, 2009, 2010). Según Castells, nos encontramos en plena época de cambio paradigmático en el mundo de la comunicación masiva, y la llamada "web social" o "Internet 2.0" - o "3.0"— son claros sintomas de ello. El paradigma comunicativo entrante, como el anterior, sigue siendo "masivo" en tanto que puede potencialmente llegar a una audiencia global. Sin embargo:

"Se trata de auto-comunicación porque la producción del mensaje es auto-generada, la definición de los potenciales receptores es auto-dirigida, y la recuperación de mensajes específicos o de contenidos de la Red Informática Mundial y de las redes de comunicación electrónica es auto-selectiva" (Castells, 2009: 55).

La socióloga Imma Tubella (2004) entiende que estos cambios paradigmáticos en el terreno de la comunicación masiva afectan al proyecto occidental de construcción de identidad. En primer lugar, el proceso de conformación del self se torna dependiente del acceso a formas virtuales de comunicación. Ello implica un problema teórico importante para las posiciones modernistas o instrumentalistas: ¿hasta qué punto pueden los poderosos "hacedores de identidad" hacer llegar su mensaje eficientemente a una población que se ve forzada día tras día a tomar decisiones en relación a su consumo de información? En segundo lugar, las narraciones sobre los endogrupos y los exogrupos entran en una fase de reelaboración permanente como consecuencia de la multiplicación de sus narradores. Se impone entonces la necesidad de conocer quién es el narrador y qué es lo narrado para comprender las dinámicas emergentes de formación de identidad.

\subsection{Las comunidades de las redes como lazos narrativos}

¿Por qué no probar de desplazarnos de la complejidad -las partes- hacia la simplicidad -el todo- en vez de hacer lo contrario?

Latour, 2001: 16

Una red puede ser representada de forma gráfica mediante nodos y enlaces. Los nodos en una red pueden representar individuos, memes, 
cuadrillas, corporaciones, estados, macro-regiones y un largo etcétera. De tal modo, la red es una metonimia de la Estructura Social capaz de satisfacer perspectivas epistemológicas muy diversas. En una red llamamos comunidades o clústeres a aquellos grupos de nodos densamente interconectados entre sí. Gu et al. (2014) afirman que la mayoría de las comunidades incrustadas en una red social virtual se constituyen, incluso si se persigue lo contrario, bajo los parámetros de lo que ellos denominan la "tentación homofílica". Se trata de un concepto anclado en la sociología de Paul F. Lazarsfeld y Robert K. Merton (1954), que consiste en la predisposición del sujeto a establecer relaciones más densas con sus grupos de iguales -ya sea en términos religiosos, educativos, laborales, axiológicos, etcétera - en vez que de sujetos que planteen cierto grado de heterogeneidad. Si contamos con los datos necesarios, podemos extraer de, o identificar en redes, comunidades mediante procedimientos matemáticos, reduciendo el potencialmente infinito número de enlaces de un nodo a una cifra cómoda y con altas capacidades descriptivas en términos de homogeneidad intra-grupal y de heterogeneidad inter-grupal (Monsalve Moreno, 2008).

Tal y como observa el decano de la Teoría del Actor-Red Bruno Latour (2005, 2011), se puede considerar que los procesos de agrupación de nodos en una red implican una reducción significativa de los enlaces que pueden caracterizar a cada uno de ellos. Es decir, al tomar en consideración solamente algunos de los enlaces que vinculan dos o más nodos, se olvidarán necesariamente otros enlaces que vinculan los mismos nodos con otros pertenecientes a otra comunidad o clúster, puesto que el procedimiento de agrupación imputa menor relevancia a dichos enlaces. Tomando el individuo como ejemplo, cabe entender que su inherente carácter poliédrico se verá desmerecido en aras de una visión agregada del mundo social —algunos de sus contactos serán invisibilizados mientras que otros van a ser resaltados-. Tal proceso implica una simplificación de la realidad social en tanto que ningún individuo es tan simple como una sola de sus afiliaciones grupales. Dicho de otra manera, lo que caracteriza la identidad de cada individuo "es su complejidad única e irrepetible, inconfundible con la de ningún otro" (Maalouf, 2001: 21).

Desde la perspectiva del paradigma de la Identidad Social (Tajfel y Turner, 1979; Tajfel, 1974; Turner et al., 1994) se considera que la "saliencia" de una categoría de identidad que opera a cierto nivel de 
abstracción hará mermar la capacidad de otras categorías pertenecientes al mismo individuo para orientar su comportamiento en una situación comparativa determinada (Abrams y Hogg, 2001). Es decir, la activación operativa y con consecuencias reales en el comportamiento del individuo de una categoría identitaria particular, como por ejemplo la identidad nacional, impide que otras categorias como la identidad religiosa, de clase, generacional, o cualquier otra oriente la acción social en una interacción. La (con-)fusión absoluta entre individuo y categoría grupal se conoce en el seno del paradigma de la Identidad Social como "proceso de despersonalización", según el cual el individuo deviene virtualmente reemplazable por cualquier otro miembro del grupo y se relacionará con los demás individuos solamente en términos de su afiliación grupal (Turner, 1999). Un buen ejemplo de este tipo de relaciones son las que mantienen dos soldados de bandos opuestos en plena batalla (Tajfel y Turner, 1979).

La simplificación de la realidad social en un Análisis de Redes Sociales es efectuada por un observador externo en vez de por el propio individuo. Cabe entender que la extracción de comunidades, si es matemáticamente significativa, tiene la capacidad de reflejar patrones comunicativos reales que coexisten en una red social. El sociólogo Klaus Eder (2009) Ilama "identidades colectivas" a los lazos narrativos que emergen de algunas redes de comunicación indirectas. Las identidades colectivas son metáforas que cristalizan algunas relaciones sociales incrustadas en redes sociales. Son internamente contradictorias e inestables, igual que lo es la Estructura Social enredada que da lugar a ellas, y que se expande, contrae y muta constantemente.

Los nodos de una red comunicativa están narrativamente entrelazados. Aunque no todas las comunidades de una red de comunicaciones indirectas llegarán a constituir categorías de afiliación poderosas en tanto que orientadoras de acción social, todas ellas se pueden entender como lazos narrativos que emergen de la interacción entre los nodos. Existen lazos narrativos de diferente naturaleza e intensidad, y bajo los términos de Eder cabe entender que muy pocos de ellos, solamente los más intensos, densos, constantes y vinculantes, tienen la capacidad de generar identidades colectivas. Según los protocolos metodológicos implementados -descritos en la siguiente sección para el análisis empírico de esta instancia comunicativa- los lazos narrativos emergentes de las interacciones que se dan en la red reflejarán vínculos 
más estables o más intermitentes, más fuertes o más circunstanciales. En cualquier caso, los procesos de agrupación permiten observar cómodamente los fenómenos de agregación surgidos de la interacción entre nodos, plasmados en distintas comunidades. Pero no hay que olvidar que los nodos están siendo agrupados al margen del valor o las características que éstos otorgan a sus enlaces. Su más o menos frecuente interacción los hará caer dentro de una u otra comunidad, y esto va a contribuir a desdibujar el resto de sus interacciones $y$, por lo tanto, parte de su identidad.

\subsection{Minar Twitter como estrategia para desenredar la realidad social enredada}

El concepto de "red social" podría ser aplicado incluso a las formas de organización de las primeras comunidades humanas. Pero su emplazamiento virtual y horizontal pertenece intensivamente a la era de la auto-comunicación masiva, de la cual la "web social" es una de sus expresiones más relevantes (Castells, 2009). Las llamadas "redes sociales virtuales" (e.g. Twitter, Facebook, LinkedIn, Academia.edu, etc.) permiten comunicaciones indirectas entre individuos de un modo que está cambiando nuestras formas de comunicación por lo que respecta a suministro, emisión, recepción y mensaje (Stassen, 2011).

En términos de la teoría del Actor-Red (Latour, 2005), se puede argumentar que las redes sociales virtuales constituyen espacios privilegiados para el "ensamblaje social". Es el elemento no humano el que facilita y dispone las relaciones entre individuos $-y$ colectivos - y el que emplaza la identidad de los usuarios de las redes sociales. La pericia técnica es un elemento imprescindible para la construcción un autoconcepto positivo en las redes sociales. La habilidad para establecer y rechazar contactos en función de los cuales los demás nos verán de una forma u otra, el buen manejo de los tiempos de publicación de los contenidos o el uso apropiado de etiquetas y palabras clave son factores que pueden aupar o derrumbar la popularidad de un usuario (Osborne, 2012).

De entre todas las redes sociales virtuales que hoy existen, el análisis de las comunicaciones en Twitter se presenta como una estrategia particularmente interesante. La popularidad de la plataforma facilita la obtención de grandes conjuntos de datos participados por miles o incluso millones de usuarios distintos. La gente utiliza Twitter 
diariamente para comunicar información cotidiana, chatear y discutir, compartir información e informar sobre noticias de todo tipo (Java et al., 2007). Según la propia empresa de comunicación, cada mes participan en Twitter 288 millones de usuarios activos, y cada día se envían 500 millones de mensajes (Twitter, 2015). Además, Twitter es una plataforma cada vez más presente en protestas y movilizaciones en todo el mundo (Hands, 2011), por lo que su encaje para el estudio de los fenómenos aquí implicados es aún mayor.

La estructura comunicativa de Twitter resulta atractiva porque permite la articulación de técnicas de Análisis de Redes Sociales cómodamente. Los mensajes o tweets tienen un máximo de 140 caracteres. El texto de los mensajes suele incorporar con frecuencia menciones hacia otros usuarios (i.e. @usuario) y suele estar ubicado bajo una etiqueta identificativa o hashtag (i.e. \#tema) al que los usuarios acuden para "tuitear". Todos estos elementos hacen sencillo el encuentro entre multitud de usuarios en torno a un tema, así como su traducción al lenguaje que requieren los programas de Análisis de Redes Sociales.

Dada la intención de este artículo (i.e. identificar y comparar algunos de los agentes que contribuyen al proceso de configuración de las identidades políticas soberanistas en el País Vasco y en Cataluña) las siguientes son las ventajas del análisis planteado:

- La popularidad de Twitter convierte esta plataforma en la elección de muchos individuos. Es posible acceder a cuantiosos conjuntos de datos altamente representativos de forma inusualmente cómoda;

- Las redes comunicativas de Twitter pueden ser artificialmente reconstruidas en redes analíticas a partir de distintos programas gratuitos y de código abierto;

- Muchas de las limitaciones de los métodos tradicionales de muestreo son superadas. Los conjuntos de datos accesibles siempre son de dominio público y proporcionan información sobre las reacciones reales de los nodos ante una situación comunicativa determinada;

- A pesar de sus limitaciones - comentadas a continuación- el análisis de movimientos sociales y dinámicas identitarias a través 
de Twitter plantea un enfoque metodológico innovador que puede ser complementado con estrategias alternativas;

- Por último, cabe mencionar que la asimetría de las relaciones en Twitter (i.e. seguir o mencionar a alguien no implica reciprocidad) permite observar la influencia o la popularidad de ciertos nodos de una manera más sofisticada que en otras redes sociales virtuales como por ejemplo Facebook, que fuerza la simetría en las relaciones de amistad entre usuarios.

Del mismo modo, las siguientes limitaciones deben ser tomadas en consideración:

- Participar en Twitter no es la elección de cada participante de la cadena humana o de la jornada de castillos humanos, ni mucho menos de todas las personas que configuran los espacios identitarios soberanistas catalán y vasco. En este sentido, no es técnicamente posible superar el sesgo de selección de la muestra dependiente del acceso a formas virtuales de comunicación;

- Los conjuntos de datos son representativos solamente para las comunicaciones analizadas. Su generalización a la realidad social offline es arriesgada y debiera ser complementada con otros métodos de investigación. Las conclusiones del presente estudio son, entonces, necesariamente provisionales;

La calidad de la muestra depende, en última instancia, de la porción de información facilitada por Twitter. El acceso a los conjuntos de datos siempre es limitado por razones de volumen, configuración de privacidad e intereses empresariales (Kelley, Sleeper y Cranshaw, 2013).

\section{ANÁLISIS DE LAS Redes SOCIALES}

Las comunicaciones en Twitter -bajo una etiqueta determinada y siendo los nodos los usuarios y las menciones los enlaces entre nodosse pueden expresar analíticamente en forma de redes sociales mediante el programa Gephi ${ }^{2}$. Las relaciones entre usuarios se pueden abordar en términos de grado ponderado, Centralidad de Grado Ponderado,

\footnotetext{
2 Gephi es un programa de código abierto y gratuito para el análisis y la visualización de redes sociales. A diferencia de otros programas más orientados a proporcionar indicadores sociológicos (e.g. UCInet), Gephi está preparado para trabajar con conjuntos de datos de un tamaño mucho mayor, lo cual lo convierte en el candidato ideal para trabajar con datos provenientes de las redes sociales virtuales.
} 
Centralidad de Intermediación y Modularidad. Seguidamente se van a introducir las nociones necesarias para entender los datos y los distintos procedimientos que se van a implementar mediante técnicas de Análisis de Redes Sociales ${ }^{3}$.

\subsection{Centralidad}

El número de enlaces de un nodo es su "grado". En otras palabras, "el grado de un nodo es el número de nodos adyacentes al mismo" (Wasserman y Faust, 1994: 100). Los grafos de red son "ponderados" cuando consisten en tres conjuntos de información distintos: un conjunto de nodos, un conjunto de enlaces y un conjunto de pesos o valores atribuidos a los enlaces (Ibid.: 140). Factores como el volumen de menciones hacia un nodo, de llamadas telefónicas, de transferencias bancarias y un largo etcétera pueden otorgar un peso determinado a un enlace. Cuando un grafo es ponderado, el grado de sus nodos tiene que ser calculado considerando el peso de sus enlaces. El grado de un nodo nos informa sobre su centralidad en la red; sin embargo, veremos que puede ser adecuada la consideración de medidas adicionales.

Los grafos de red pueden ser dirigidos o no dirigidos. Cuando no hay distinción entre el origen y el destino de los enlaces entre nodos estos pueden representarse en un grafo no dirigido (Ibid.: 96). Por ejemplo, las relaciones de amistad en Facebook requieren conformidad mutua y son simétricas incluso si no lo es la misma relación en el mundo offline. En cambio, cuando los enlaces entre nodos tienen origen y destino, estos pueden representarse en un grafo dirigido (Ibid.: 122-123). Las menciones entre usuarios en Twitter son casi siempre ponderadas y siempre dirigidas en tanto que no exigen reciprocidad aunque esta pueda existir. El grado de un nodo en un grafo dirigido puede ser desglosado entre "grado de entrada" y "grado de salida", mientras que esto es imposible en un grafo no dirigido (Hanneman y Riddle, 2005: 97).

La centralidad de un nodo depende de una suerte de propiedades derivadas de su posición en la red. Los nodos altamente centrales pueden ser interpretados como prominentes, influyentes, líderes, prestigiosos, populares, poderosos, y un largo etcétera (Borgatti, Everett y Johnson, 2013: 164). Sea como fuere, se puede asumir que los nodos

\footnotetext{
${ }^{3}$ Para profundizar en el tema se recomienda la lectura de Wasserman y Faust (1994) o de Hanneman y Riddle (2005).
} 
altamente centrales se encuentran en posiciones estructurales favorables en el seno de la red (Hanneman y Riddle 2005: 145). Pero la centralidad de los nodos puede ser utilizada para analizar, además de las posiciones estructurales particulares, las características de la red como un todo.

El sociólogo matemático Linton C. Freeman, padre del programa UCInet para el Análisis de Redes Sociales, introdujo tres formas de medir la centralidad de un grafo. Aquí solamente se considerará la Fórmula General para la Centralización de Grado (Freeman, 1978). Se trata de una medida de la varianza de la desigualdad en una red social en comparación con una red idealmente horizontal del mismo tamaño y densidad:

$$
C_{D}=\frac{\sum_{i=1}^{n}\left[C_{D}\left(p^{*}\right)-C_{D}\left(p_{i}\right)\right]}{[(P-1)(P-2)]}
$$

El $\left\{C_{D}\left(p_{i}\right)\right\}$ en el numerador representa el grado de un nodo cualquiera, mientras que el $\left\{C_{D}\left(p^{*}\right)\right\}$ representa el grado más alto de la red. El índice en el denominador se refiere a la suma máxima de diferencias $\left\{\max \sum_{i=1}^{n}\left[C_{D}\left(p^{*}\right)-C_{D}\left(p_{i}\right)\right]\right\}$ y es determinada por $\{(P-1)(P-2)\}$, siendo $P$ el tamaño de la muestra (Ibid.: 228-229). La Centralidad de Grado $\left\{C_{D}\right\}$ variará de 0 a 1 , y será de 1 si un solo nodo está perfectamente conectado con el resto y el resto sólo interactúa con él. En grafos dirigidos, los resultados tienen que separarse entre Centralidad de Grado de Entrada $\left(C_{D}^{\text {in }}\right)$ y Centralidad de Grado de Salida ( $C_{D}^{\text {out }}$ ) (Wasserman y Faust, 1994: 180-181).

Los grados ponderados de entrada y de salida de un nodo pueden no aportar toda la información necesaria para interpretar su centralidad. Por ejemplo, la posición geodésica de un nodo puede afectar su situación estructural en términos de influencia o de poder (Hanneman y Riddle, 2005: 163). La Centralidad de Intermediación es una medida sobre "cuán habitualmente un nodo cae en medio del patrón de comunicación más corto entre dos otros nodos" (Borgatti, Everett y Johnson, 2013: 174). La medida se basa en la asunción de que el nodo que preside un cuello de botella comunicativo será más poderoso (Tsvetovat y Kouznetsov, 2011: 51). La influencia o el poder derivado de la Centralidad de Intermediación de un nodo puede sostenerse de forma complementaria o no con un grado de entrada significativamente alto, puesto que se trata de características diferentes y de propiedades distintas de la posición estructural de un nodo. 
La Centralidad de Intermediación de un nodo (e.g. el nodo j) se puede calcular con la siguiente fórmula:

$$
b_{j}=\sum_{i<k} \frac{g_{i j k}}{g_{i k}}
$$

El $\left\{g_{i j k}\right\}$ en el numerador es el número de enlaces geodésicos entre los nodos $i$ y $k$ que contiene el nodo $j$. El $\left\{g_{i k}\right\}$ en el denominador expresa el total de patrones geodésicos entre $i$ y $k$. Las proporciones son sumadas para cada par de nodos en relación al nodo $j$ y se obtiene un valor unitario que constituye la Centralidad de Intermediación del nodo en cuestión $\left\{b_{j}\right\}$ (Freeman, 1978: 223). El algoritmo utilizado por Gephi para calcular la Centralidad de Intermediación de cada nodo está basado en la fórmula original de Freeman (Brandes, 2001).

\subsection{Detección de comunidades}

En términos generales, la detección de comunidades en una red es posible cuando la mayoría de los nodos no están conectados entre sí y la densidad de la red es baja (Hanneman y Riddle, 2005: 122). Mediante las pruebas de modularidad obtendremos información sobre la calidad de las comunidades identificadas en una red en su conjunto. En este artículo se va a utilizar el algoritmo de detección rápida de comunidades de Blondel et al. (2008), que está diseñado para grafos dirigidos y puede ser implementado con el software Gephi. El algoritmo asume la fórmula de Modularidad para redes ponderadas de Mark Newman (2004), que indica la calidad de una partición de red:

$$
Q=\frac{1}{2 m} \sum_{i j}\left[A_{i j}-\frac{k_{i} k_{j}}{2 m}\right] \delta\left(c_{i}, c_{j}\right)
$$

El número de enlaces en una red es establecido mediante $\left\{m=1 / 2 \sum_{i j} A_{i j}\right\}$, y el $\left\{k_{i} k_{j} / 2 m\right\}$ tiene que ver con la probabilidad de que un enlace exista entre dos nodos. El $\left\{c_{i}\right\}$ es la comunidad en la que el nodo $i$ es asignado. A través de la función $\{\delta\}$ se compara la fracción de enlaces que contiene una comunidad con la misma comunidad bajo asignación aleatoria de los enlaces de la red. Los valores de modularidad $\{Q\}$ cercanos a cero indicarán una partición de mala calidad. El valor máximo de $\{Q\}$ es \pm 1 . Valores positivos de 0.3 o más indican la buena calidad de la partición del grafo en comunidades (Ibid., 2004: 7). Nunca se obtendrán cifras negativas porque el algoritmo de detección comunitaria utilizado por Gephi las impide. 
El algoritmo de detección comunitaria de Vincent D. Blondel et al. (2008) se estructura en dos etapas repetidas iteradamente. En primer lugar, cada nodo es determinado como una comunidad -en la partición inicial $(t=0)$ hay tantas comunidades como nodos-. Los nodos se asocian progresivamente de manera aleatoria y la ganancia positiva en Modularidad es permanentemente evaluada. Si una asociación entre nodos no supone una ganancia en Modularidad, el nodo permanece en la comunidad original. El proceso acaba cuando el máximo local de Modularidad positiva es alcanzado. Seguidamente se diseña una nueva red cuyos nodos son las comunidades halladas en la etapa anterior. Los pesos de los enlaces entre nodos se establecen sumando los pesos de los enlaces originales. Ambos pasos se repiten hasta que no pueden efectuarse cambios beneficiosos en términos de Modularidad (Ibid., 2008: 4-5).

El procedimiento estructura de manera jerárquica las comunidades encontradas. A cada nivel de asociación le corresponderá una cifra determinada de Modularidad, siendo ésta más problemática en comunidades pequeñas (Fortunato y Barthélemy, 2007). Gephi requiere la combinación del algoritmo anterior con la determinación manual del límite de resolución del procedimiento. El límite de resolución es el tiempo de iteración del algoritmo. Mientras que la partición óptima a $t=0$ será de tantas comunidades como nodos, obtendremos macrocomunidades bajo largos periodos de iteración (Lambiotte, Delvenne y Barahona, 2009). No existe una norma establecida para determinar la resolución óptima del procedimiento, ya que ésta depende de factores como la cantidad de nodos o de enlaces en la red. Para obtener una partición de la red aceptable en términos de cifras de Modularidad se recomienda implementar el algoritmo más de una vez y bajo resoluciones distintas, escogiendo aquella partición con mejores cifras (Seifi et al., 2013).

\section{MÉTOdO}

El siguiente análisis se basa en las etiquetas \#BasquesDecide y \#Up4Freedom. Ambas tienen una orientación internacional -a diferencia de \#GureEskuDago- y un número similar de participantes - a diferencia de \#CatalansWantToVote, que registró una participación cuatro veces mayor-. Además, en contraste con las dos etiquetas excluidas, se trata de etiquetas expresamente creadas para la actividad planteada, cuya relevancia fue mucho más breve en el tiempo. 


\subsection{Diseño}

La etiquetas \#BasquesDecide y \#Up4Freedom fueron monitorizadas desde el 7 hasta el 17 de junio de 2014 mediante la ya extinta aplicación ScraperWiki, que permitía la creación de una base de datos con gran parte de los tweets referentes a un hashtag o palabra clave durante un periodo temporal determinado. Durante estos diez días, 21.230 mensajes de 8.421 usuarios distintos fueron registrados en el caso de \#BasquesDecide y 22.041 mensajes de 7.536 usuarios diferentes en el caso de \#Up4Freedom. Un total de 317 mensajes que se encontraban en los dos conjuntos de datos (i.e. mensajes que incluian las etiquetas \#BasquesDecide y \#Up4Freedom) fueron eliminados del conjunto \#Up4Freedom y mantenidos en el otro conjunto de datos para eliminar su sobrerepresentación en el grafo de red combinado - no fueron imputados en sentido inverso simplemente para no incrementar la diferencia entre el número de mensajes entre ambos conjuntos de datos-.

Posteriormente, los datos fueron refinados mediante Google Refine: se extrajeron las menciones de los mensajes y se dio al conjunto un formato apropiado para Gephi basado en las columnas "emisor" y "receptor" de mención. El proceso implicó la creación de los nodos adicionales necesarios (i.e. usuarios mencionados que no mencionaron a nadie) y configuró dos conjuntos de datos con 8.255 nodos y 18.378 enlaces en el caso de \#BasquesDecide, y con 7.352 nodos y 27.989 enlaces en el caso de \#Up4Freedom. Finalmente, fueron filtrados de ambos conjuntos de datos los nodos aislados del componente gigante (i.e. los nodos virtualmente inaccesibles, puesto que no están enlazados mediante menciones con el grueso de nodos de la red) y los auto-enlaces (i.e. menciones hacia uno mismo) mediante el programa Gephi. El grafo de red para \#BasquesDecide cuenta con 8.019 nodos (el $97,14 \%$ del total) y 18.073 enlaces (98,34\%); el grafo para \#Up4Freedom cuenta con 7.143 nodos $(97,29 \%)$ y 27.668 enlaces $(98,85 \%)$; y el grafo combinado cuenta con 14.511 nodos y 45.705 enlaces.

Conforme a la literatura presentada anteriormente, y dada la naturaleza más inductiva que deductiva del planteamiento de la investigación aquí desarrollado, no van a ser lanzadas hipótesis explícitas sobre la morfología de las redes tratadas, sino que se van a explorar las diferencias entre ambos grafos y el grafo combinado en los siguientes términos:

- Centralización de grado, tanto para $C_{D}^{o u t}$ como para $C_{D}^{\text {in }}$.

- Grado ponderado de entrada y salida. 
- Centralidad de Intermediación.

- Partición del grafo y Modularidad.

\subsection{Resultados}

\subsection{1. \#BasquesDecide}

Mientras que la red está centralizada en de grado de entrada $\left(C_{D}^{i n}=0,532\right)$; está relativamente descentralizada en grado de salida $\left(C_{D}^{\text {out }}=0,278\right)$. Tal cosa indica que mencionar y ser mencionado en \#BasquesDecide son fenómenos claramente distintos. Las cifras de centralización son altas en ambos casos.

El grado ponderado medio de la red es de 2,78. Los diez usuarios con mayor grado de entrada ponderado - siendo 4.270 el más alto y 305 el más bajoson la organización convocante (@gureeskudago), cinco medios de comunicación (@naiz_info, @berria, @argia, @ahotsainfo y @eitbcomnoticias) y cuatro usuarios individuales de entre los que destacan un periodista y un político. Cabe comentar que la mayoría de los usuarios con alto grado de entrada sostienen también grados de salida elevados no hay entre ellos usuarios sin menciones salientes-. Los diez usuarios con mayor grado de salida ponderado -máximo 226 y mínimo $74-$ son la misma organización convocante y 9 usuarios individuales.

Los diez usuarios con mayor Centralidad de Intermediación son la organización convocante (@gureeskudago), cuatro medios de comunicación (@naiz_info, @berria, @ahotsainfo y @argia) y cinco usuarios individuales.

El algoritmo de detección de comunidades implementado a 1.0 puntos de resolución ha dado lugar a trece comunidades y una modularidad de 0,513 puntos positivos que denota una muy buena calidad de las particiones. Las trece comunidades están distribuidas de la siguiente manera (tabla 1):

Tabla 1. Grafo de red \#BasquesDecide particionado a 1.0 puntos de resolución

\begin{tabular}{cc}
\hline Comunidad & Porcentaje de nodos \\
\hline $\mathbf{0}$ & $26,54 \%$ \\
$\mathbf{4}$ & $17,99 \%$ \\
$\mathbf{1 0}$ & $13,44 \%$ \\
$\mathbf{3}$ & $7,62 \%$ \\
$\mathbf{6}$ & $6,97 \%$ \\
\hline
\end{tabular}




\begin{tabular}{cc}
\hline $\mathbf{9}$ & $5,81 \%$ \\
$\mathbf{7}$ & $5,36 \%$ \\
$\mathbf{2}$ & $3,72 \%$ \\
$\mathbf{1}$ & $3,19 \%$ \\
$\mathbf{1 1}$ & $3,14 \%$ \\
$\mathbf{8}$ & $2,74 \%$ \\
$\mathbf{5}$ & $2,71 \%$ \\
$\mathbf{1 2}$ & $0,76 \%$ \\
\hline
\end{tabular}

Fuente: Diseño basado en los datos obtenidos y refinados

Los siguientes grafos de red (figuras 1 y 2) muestran las 13 comunidades identificadas de forma conjunta y separada. El tamaño de los nodos ha sido determinado según su Centralidad de Intermediación. Los grafos se han diseñado mediante el algoritmo de visualización Force Atlas 2 (Jacomy et al., 2014). Éste se ha implementado considerando el peso de los enlaces, una gravedad de 1,0 puntos, una aproximación de 1,2 y la opción de aproximar repulsión activada, por lo que los nodos se agrupan y separan en función de la densidad de sus enlaces. Después del procedimiento se ha activado la prevención de superposición entre nodos para mejorar las capacidades visuales del grafo; de forma simultánea, se ha aumentado la gravedad y se ha reducido la escala del grafo el para acercar los nodos menos conectados con el componente gigante, cosa que da lugar a un gráfico visualmente cohesionado.

Entre los nodos con más Centralidad de Intermediación en la comunidad 0 nos encontramos con usuarios relativos a la organización Gure Esku Dago (e.g. @gureeskudago, @gureeskudima, @gureeskuerandio, etc.) con campañas virtuales (e.g. nabarraconb) e incluso con algún grupo musical vascoparlante. El nodo más prominente de la comunidad y de la red, cuyo tamaño es significativamente superior a los demás, es la organización convocante.

En contraste, en la comunidad 4 destacan por su importancia usuarios relativos a medios de comunicación (e.g. @naiz_info, info7irratia, @garanet, etc.). También son muy importantes usuarios individuales que hacen un uso profesional de Twitter, como políticos, periodistas o formadores. Su importancia visual es muy menor a la de @gureeskudago. La comunidad 10 está cortada por el mismo patrón que la 4 (@berria, @argia, @uriolainfo, @ hamaikatb, etc.) aunque alguna sección local de Gure Esku Dago también aparece como prominente (e.g. @gedarrasate). 


\section{Figura 1. Comunidades detectadas en el grafo de red \#BasquesDecide}

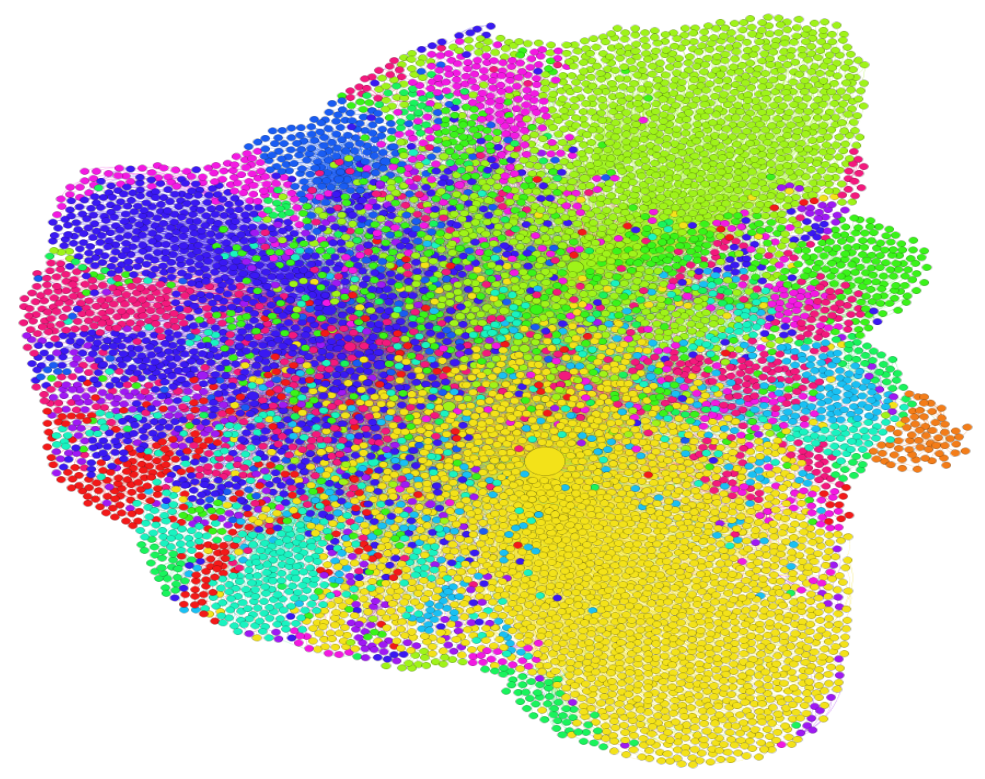

Fuente: Diseño basado en los datos obtenidos y refinados

Figura 2. Comunidades detectadas en el grafo de red \#BasquesDecide separadas

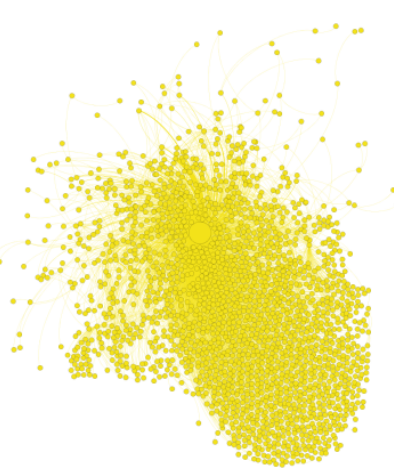

Comunidad 0

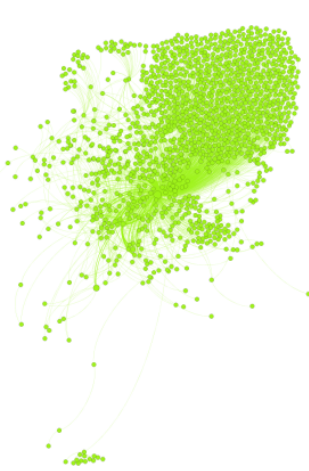

Comunidad 4

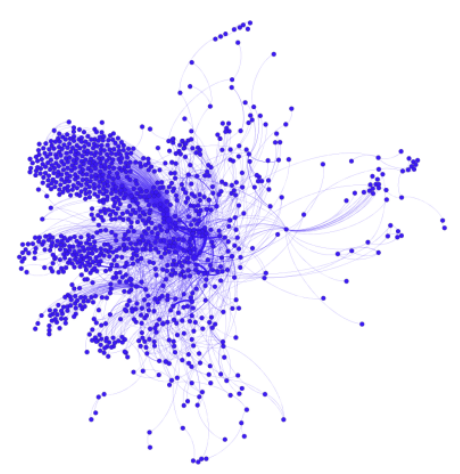

Comunidad 10

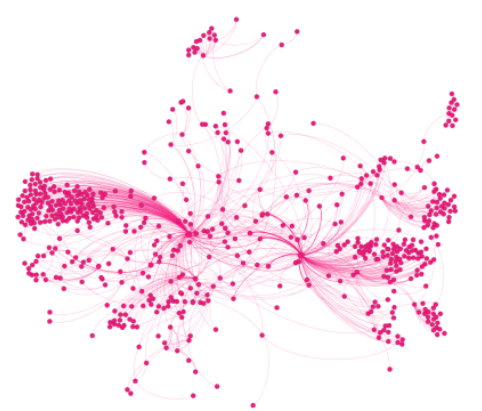

Comunidad 3

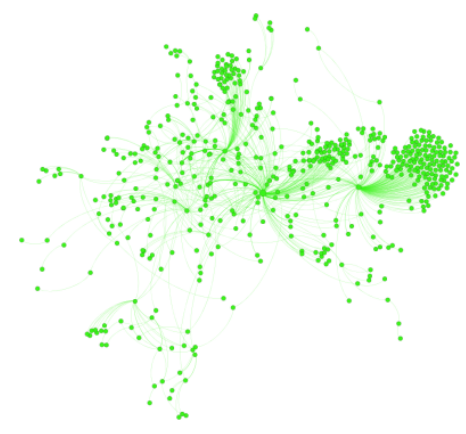

Comunidad 6

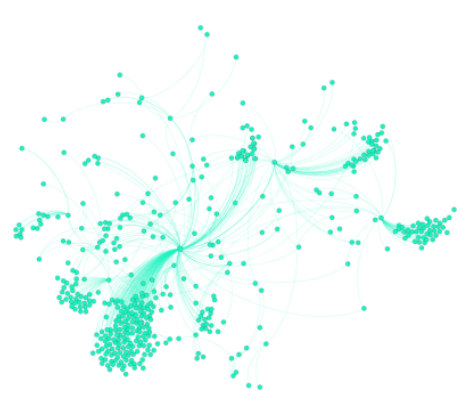

Comunidad 9

Papeles del CEIC 


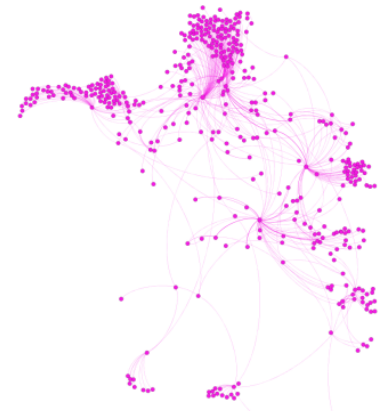

Comunidad 7

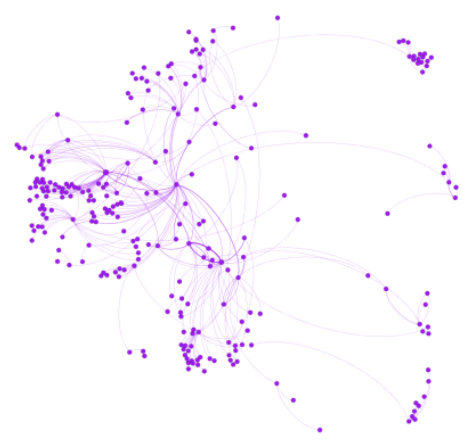

Comunidad 11

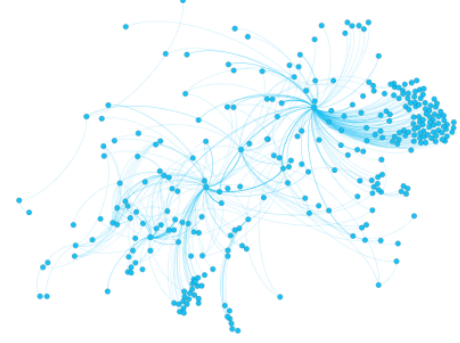

Comunidad 2

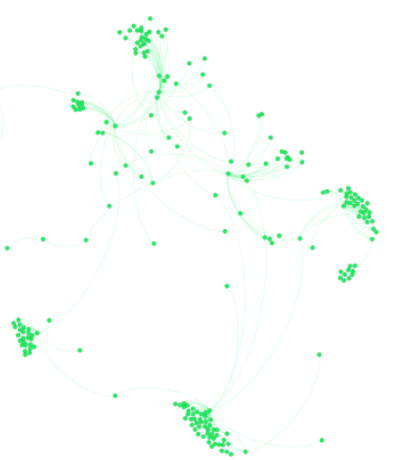

Comunidad 8

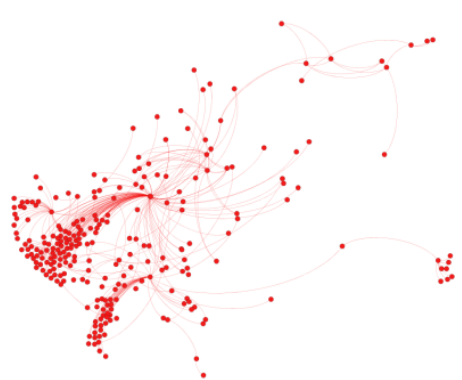

Comunidad 1

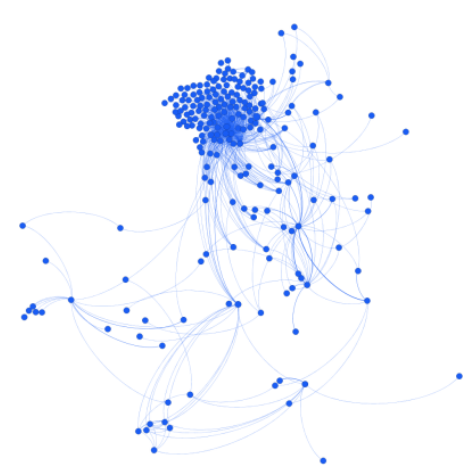

Comunidad 5

\section{Comunidad 12}

Fuente: Diseño basado en los datos obtenidos y refinados

La comunidad 3 es la primera en importancia donde sus nodos más centrales en términos de intermediación son usuarios individuales. Por ello, se puede afirmar que la importancia en la red de nodos relativos a organizaciones y medios de comunicación es muy elevada. Estos usuarios vinculan a los demás entre si con mucha fuerza y mantienen unida la red. A pesar de ello, resulta obvio que los usuarios de tipo individual son fundamentales - son los más abundantes y algunos de ellos gozan de alta centralidad de intermediación-. 


\subsection{2. \#Up4Freedom}

Se trata de una red moderadamente descentralizada en términos de grado de entrada $\left(C_{D}^{i n}=0,232\right)$ y muy descentralizada en su grado de salida $\left(C_{D}^{\text {out }}=0,061\right)$. Es una red más descentralizada que la anterior. He aquí la primera diferencia.

El grado ponderado medio de \#Up4Freedom es de 4,477, dos puntos superior al de \#BasquesDecide. Se trata por lo tanto de una red cuyos nodos son más participativos. El nodo con mayor grado de entrada ponderado es Òmnium Cultural (@omnium), con 1.663 menciones recibidas. Entre los diez usuarios con mayor grado de entrada - siendo el más bajo de 407- también nos encontramos con tres colles castelleres (@verds, @collavella, @jovesvalls), con la sección francesa de la Assemblea Nacional Catalana (ANC) (@anc_france) y con cinco usuarios individuales.

Por lo que respecta los diez usuarios con mayor Centralidad de Intermediación, entre estos hay ocho usuarios individuales, la organización convocante y la cuenta en lengua inglesa de la ANC (@catalanassembly). El primer medio de comunicación en importancia según su Cercanía de Intermediación no aparece hasta el puesto 112 (@vilaweb_en).

El algoritmo de detección de comunidades a 1.0 puntos de resolución detectó nada menos que 21 comunidades con una modularidad de 0,434 puntos positivos. Se trata de un número moderadamente elevado de comunidades y de una muy buena calidad de la partición. En contraste con el grafo de red anterior, cabe destacar la mayor similitud entre las comunidades en su tamaño. Las comunidades quedan distribuidas de la siguiente manera (tabla 2).

Tabla 2. Grafo de red \#BasquesDecide particionado a 1.0 puntos de resolución

\begin{tabular}{cc}
\hline Comunidad & Porcentaje de nodos \\
\hline $\mathbf{8}$ & $10,62 \%$ \\
$\mathbf{1}$ & $10,28 \%$ \\
$\mathbf{1 4}$ & $8,77 \%$ \\
$\mathbf{7}$ & $8,04 \%$ \\
$\mathbf{6}$ & $7,24 \%$ \\
$\mathbf{3}$ & $6,93 \%$ \\
$\mathbf{2}$ & $6,68 \%$ \\
$\mathbf{1 0}$ & $5,51 \%$ \\
$\mathbf{0}$ & $5,24 \%$ \\
\hline
\end{tabular}




\begin{tabular}{cc}
\hline 19 & $4,67 \%$ \\
13 & $4,17 \%$ \\
$\mathbf{9}$ & $3,66 \%$ \\
15 & $3,44 \%$ \\
17 & $2,99 \%$ \\
20 & $2,59 \%$ \\
$\mathbf{4}$ & $2,57 \%$ \\
$\mathbf{1 1}$ & $2,04 \%$ \\
$\mathbf{1 6}$ & $1,47 \%$ \\
$\mathbf{1 8}$ & $1,33 \%$ \\
$\mathbf{5}$ & $0,95 \%$ \\
$\mathbf{1 2}$ & $0,81 \%$ \\
\hline
\end{tabular}

Fuente: Diseño basado en los datos obtenidos y refinados

Los siguientes grafos de red (figuras 3 y 4) muestran las 21 comunidades halladas de forma conjunta y separada. El tamaño de sus nodos ha sido determinado según su Centralidad de Intermediación, y el algoritmo y el procedimiento utilizados para el diseño del grafo ha sido el mismo que para \#BasquesDecide.

Los diez nodos más centrales en intermediación de las comunidades 8, 14, 7 y 6 son usuarios individuales. Los nodos pertenecientes a organizaciones políticas y culturales, asi como a medios de comunicación son claramente residuales en estos grupos.

El nodo más prominente en Cercanía de Intermediación de la segunda comunidad más numerosa, la comunidad 1, es Òmnium Cultural (@omnium). Entre ellos también hay un usuario perteneciente a una colla castellera (@jovesvalls). Entre los diez nodos más centrales en intermediación en la comunidad 3 también hay una agrupación de castillos humanos (@collavella) y dos secciones de la ANC (@ancfrance y @ancvallesorient). En ambos casos el grupo más numeroso es el de usuarios individuales.

De entre las comunidades que agrupan más del $5 \%$ de los nodos, solamente en la comunidad 0 hay una concentración significativa de usuarios relativos a organizaciones en posiciones relevantes por su Centralidad de Intermediación (e.g. @catalanassembly, @anc_ireland, @ancxile, @assemblea, @ancstand, @ancmongat, @assembleate, etc.). Se trata de una red donde el rol de los usuarios de tipo individual es decididamente central. En contraste con la red \#BasquesDecide, el papel 
de usuarios relativos a organizaciones o a medios de comunicación es muy inferior.

Figura 3. Comunidades detectadas en el grafo de red \#Up4Freedom

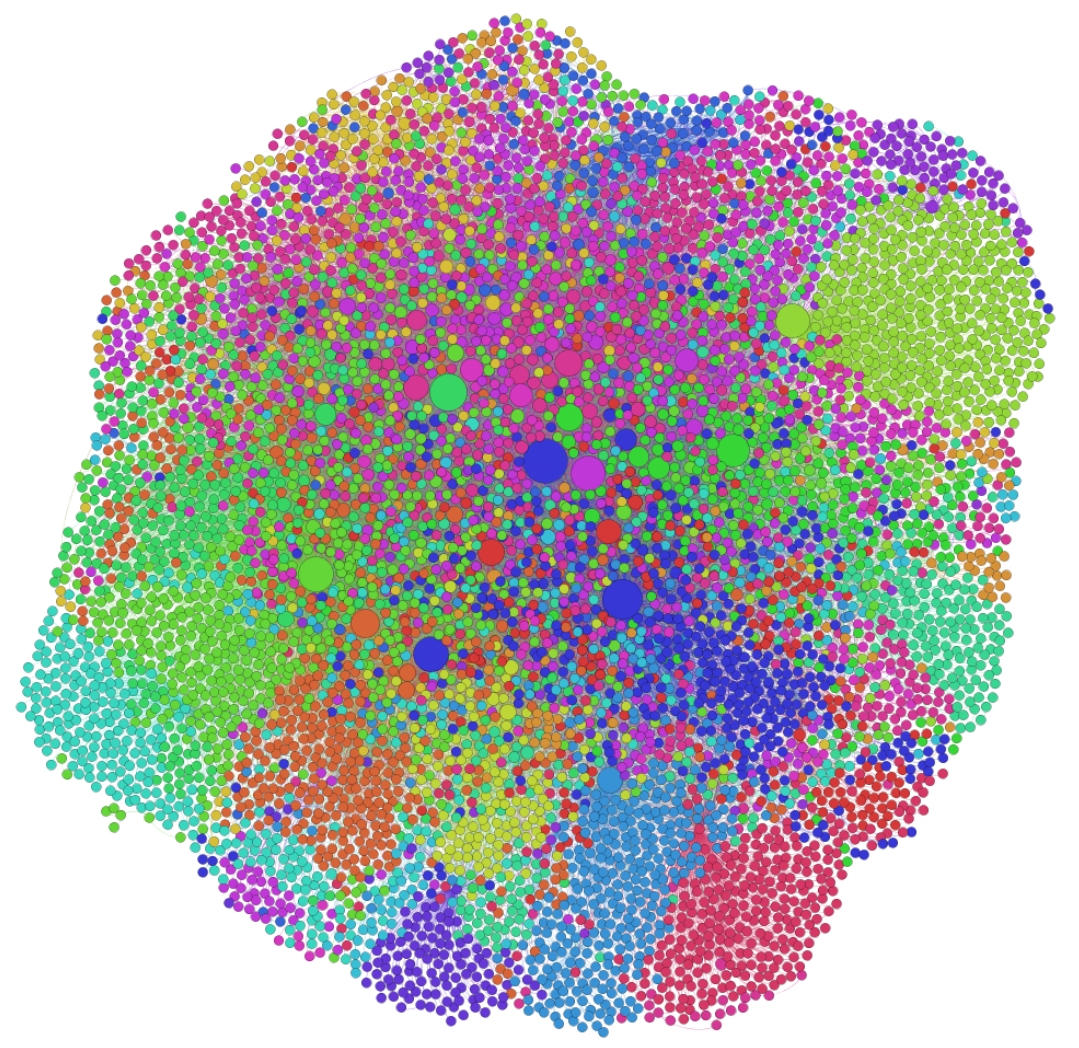

Fuente: Diseño basado en los datos obtenidos y refinados

Figura 4. Comunidades detectadas en el grafo de red \#Up4Freedom desagregadas

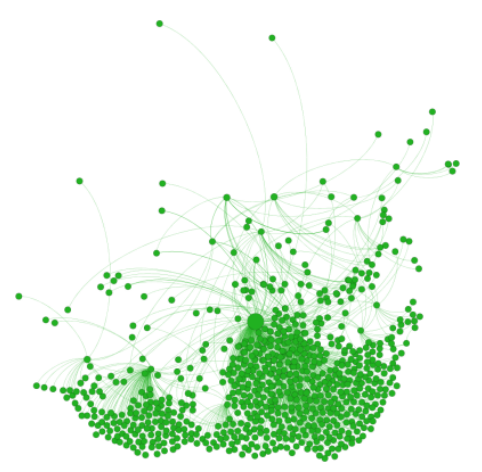

Comunidad 8

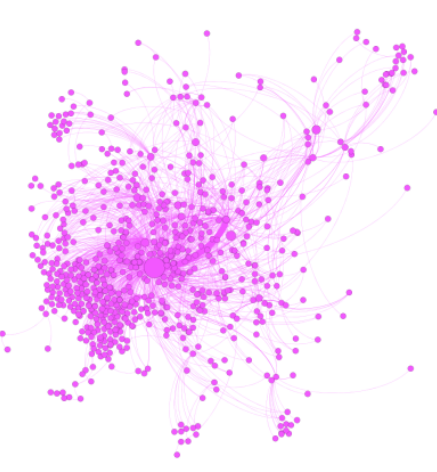

Comunidad 1

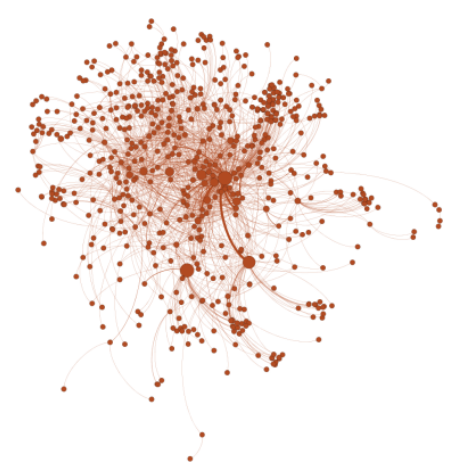

Comunidad 14 


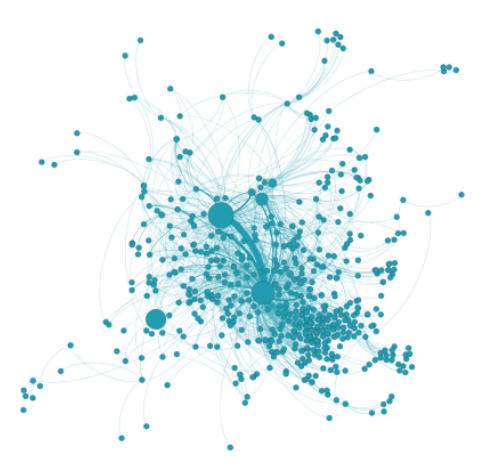

Comunidad 7

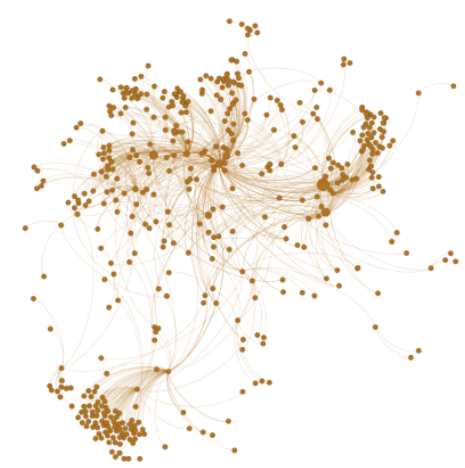

Comunidad 2

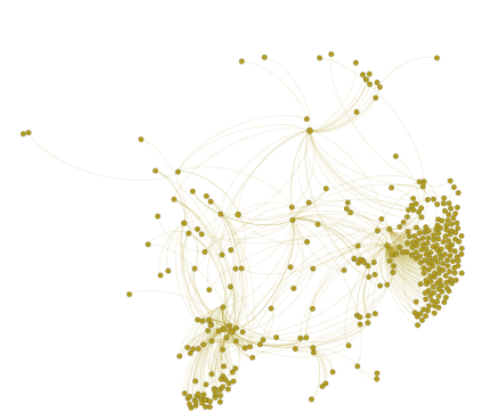

Comunidad 19

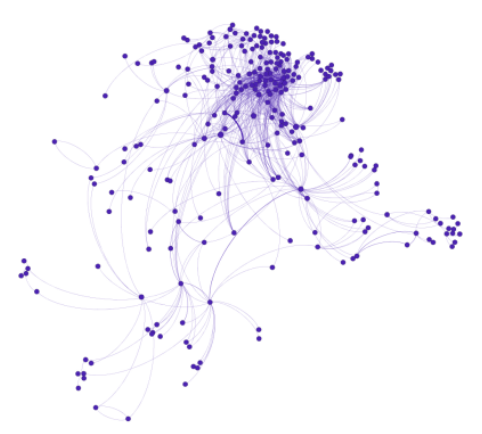

Comunidad 15

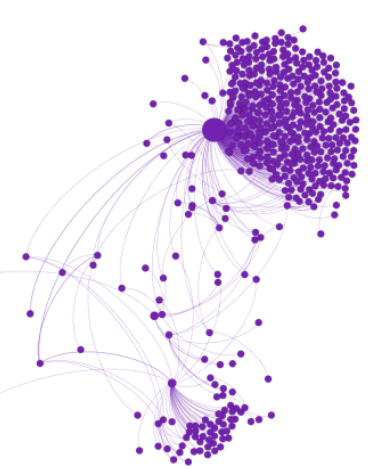

Comunidad 6
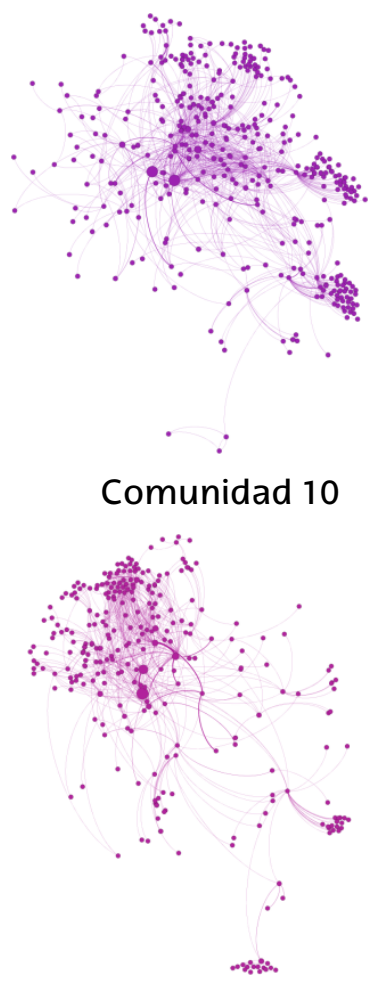

Comunidad 13

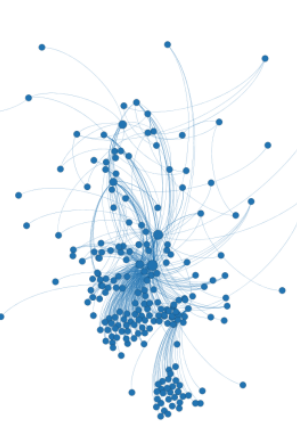

Comunidad 17

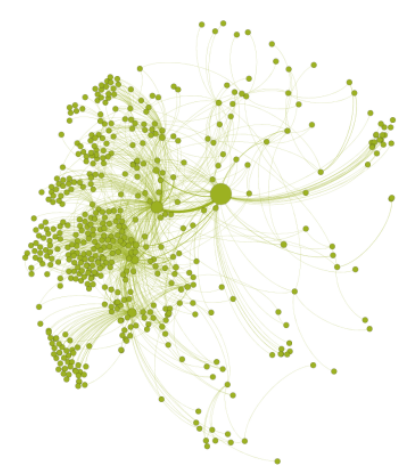

Comunidad 3

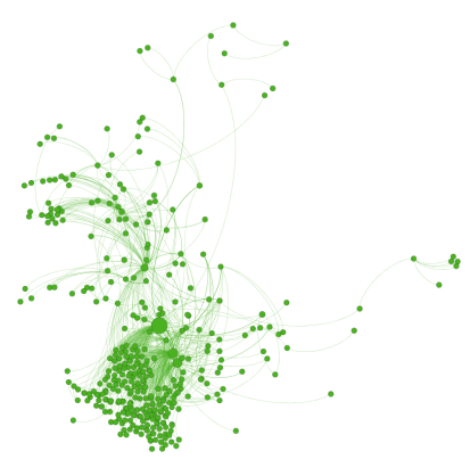

Comunidad 0

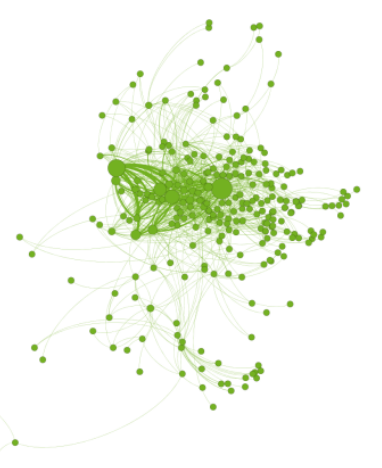

Comunidad 9

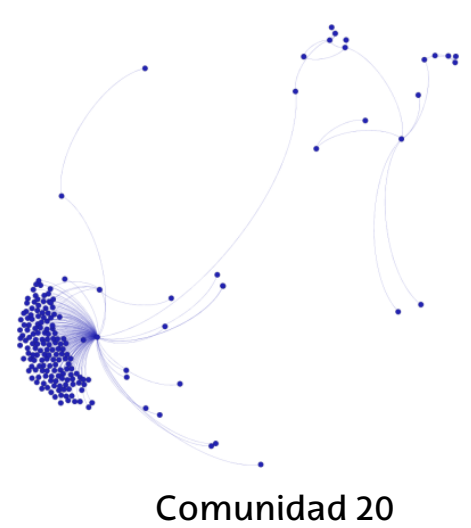

Papeles del CEIC 


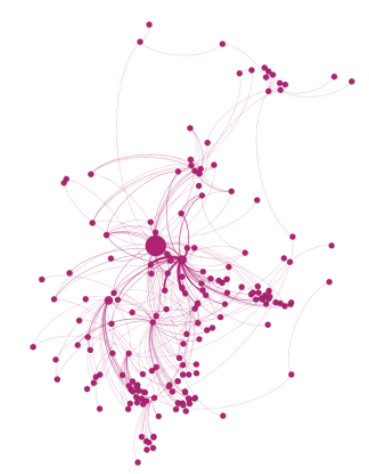

Comunidad 4

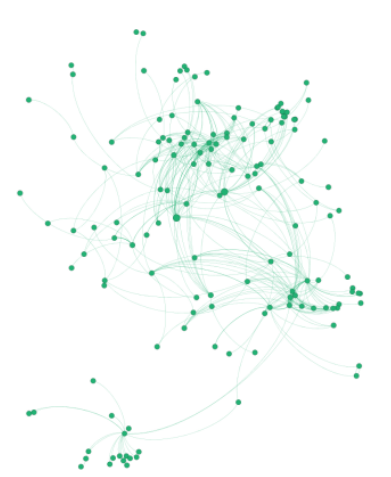

Comunidad 11

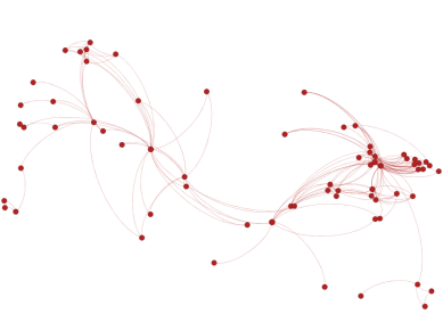

Comunidad 5

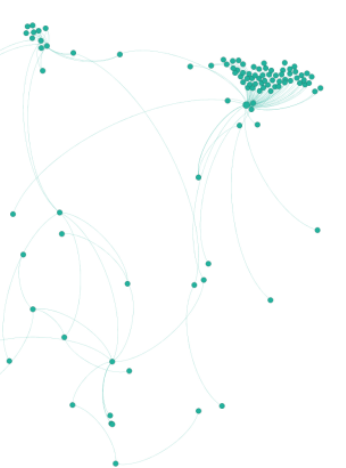

Comunidad 16

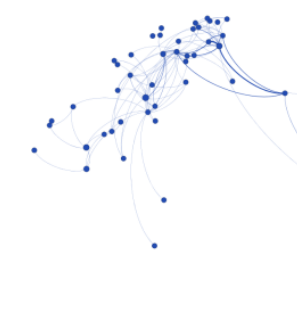

Comunidad 12

Comunidad 18

Fuente: Diseño basado en los datos obtenidos y refinados

\subsection{3. \#BasquesDecide y \#Up4Freedom}

La red resultante de la unión de ambos conjuntos de datos está moderadamente descentralizada en grado de entrada $\left(C_{D}^{i n}=0,295\right)$ y descentralizada en grado de salida $\left(C_{D}^{\text {out }}=0,030\right)$.

El grado ponderado medio de la red es de 3,745 . Los cuatro nodos con mayor grado de entrada ponderado son @gureeskudago (4.281) @naiz_info (2.043), @omnium (1.663) y @berria (959). Entre los otros seis nodos de los diez más mencionados hay otro medio de comunicación (@argia), una agrupación de castillos humanos (@verds) y cuatro usuarios individuales, dos de los cuales activos en \#BasquesDecide, y los otros dos en \#Up4Freedom.

Entre los diez usuarios que más menciones han emitido hay nueve usuarios individuales y @gureeskudago. Solamente uno de los usuarios individuales en el listado proviene del conjunto de datos extraídos en base a la etiqueta \#BasquesDecide. Ello es consecuencia directa de la menor participación de sus nodos que ya ha sido observada. 
Los diez usuarios con mayor Centralidad de Intermediación son @gureeskudago, @argia, @naiz_info, @taxistesindepes (i.e. una sectorial de la ANC) y seis usuarios individuales de los cuales solo uno proviene del conjunto de datos \#BasquesDecide.

Implementado a 1.0 puntos de resolución, el algoritmo ha dado lugar a veinte comunidades a 0,536 puntos positivos de Modularidad. Se trata de una cifra razonablemente reducida de comunidades dado el tamaño de la red (i.e. 14.511 nodos y 45.705 enlaces), y de una calidad de partición muy buena. Las comunidades quedan distribuidas de la siguiente forma (tabla 3):

Tabla 3. Grafo de red \#BasquesDecide y \#Up4Freedom particionado a 1.0 puntos de resolución

\begin{tabular}{cc}
\hline Comunidad & Porcentaje de nodos \\
\hline $\mathbf{0}$ & $29,32 \%$ \\
$\mathbf{5}$ & $17,03 \%$ \\
$\mathbf{9}$ & $14,38 \%$ \\
$\mathbf{1 1}$ & $9,08 \%$ \\
$\mathbf{6}$ & $7,64 \%$ \\
$\mathbf{1 3}$ & $6,79 \%$ \\
$\mathbf{1 0}$ & $4,74 \%$ \\
$\mathbf{1 2}$ & $2,74 \%$ \\
$\mathbf{3}$ & $2,44 \%$ \\
$\mathbf{4}$ & $1,70 \%$ \\
$\mathbf{2}$ & $1,23 \%$ \\
$\mathbf{1 7}$ & $0,99 \%$ \\
$\mathbf{1 8}$ & $0,75 \%$ \\
$\mathbf{8}$ & $0,54 \%$ \\
$\mathbf{1}$ & $0,51 \%$ \\
$\mathbf{1 9}$ & $0,03 \%$ \\
$\mathbf{1 4}$ & $0,03 \%$ \\
$\mathbf{7}$ & $0,03 \%$ \\
$\mathbf{1 6}$ & $0,02 \%$ \\
$\mathbf{1 5}$ & $0,02 \%$ \\
\hline
\end{tabular}

Fuente: Diseño basado en los datos obtenidos y refinados

En el siguiente grafo de red (figura 5) podemos observar las veinte comunidades y los nodos, cuyo tamaño ha sido determinado según su Centralidad de Intermediación. El grafo ha sido diseñado mediante el mismo sistema que los anteriores. En la figura 6 podemos observar de forma comparativa los enlaces entre nodos pertenecientes a cada 
conjunto de datos. Para ello, el tamaño de los nodos ha sido reducido y se ha desvinculado de su Centralidad de Intermediación, por lo que todos tienen el mismo tamaño.

Figura 5. Comunidades detectadas en el grafo de red \#BasquesDecide y \#Up4Freedom

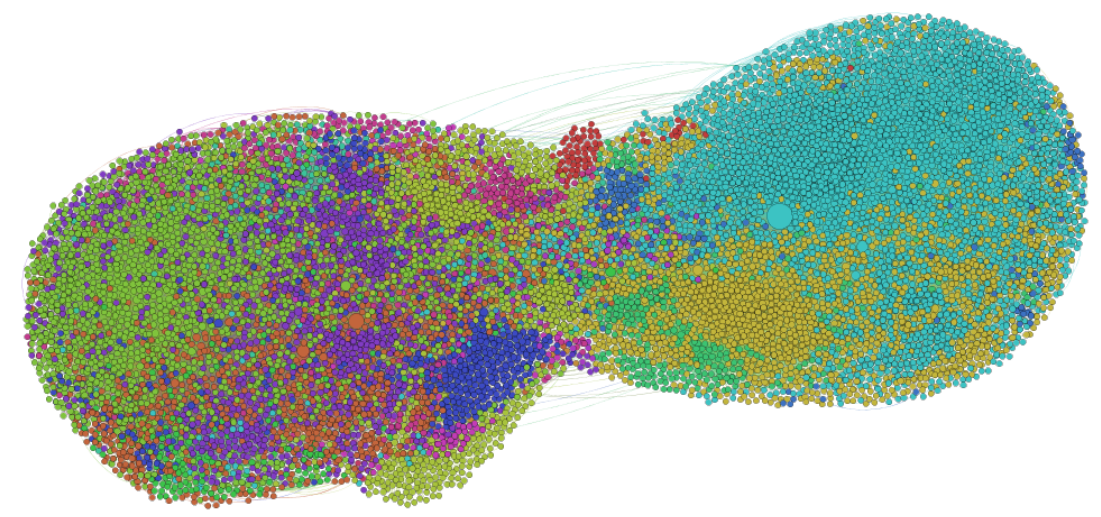

Fuente: Diseño basado en los datos obtenidos y refinados

Figura 6. Enlaces pertenecientes a \#BasquesDecide y a \#Up4Freedom según comunidad

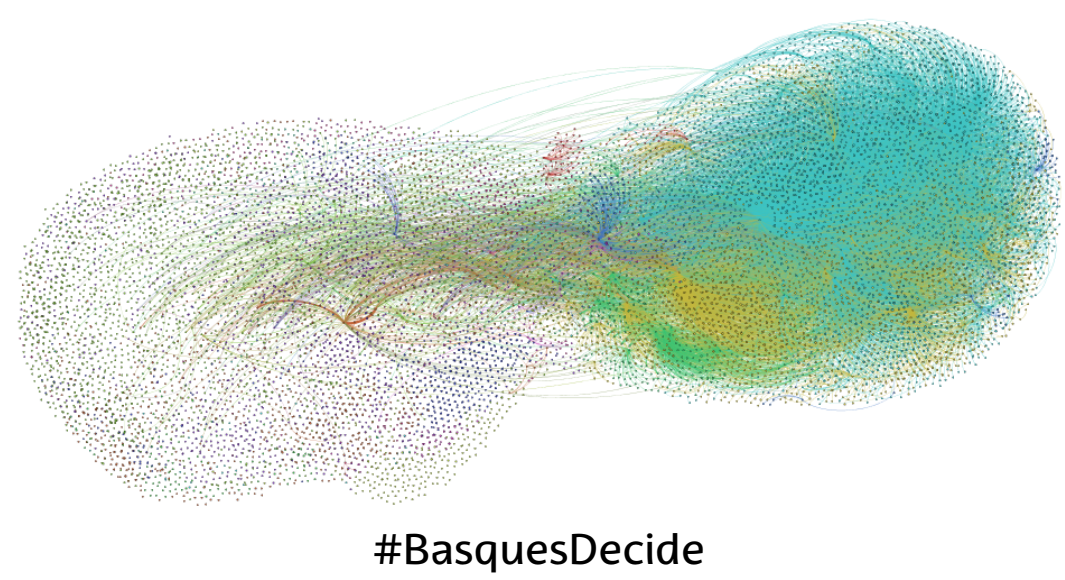




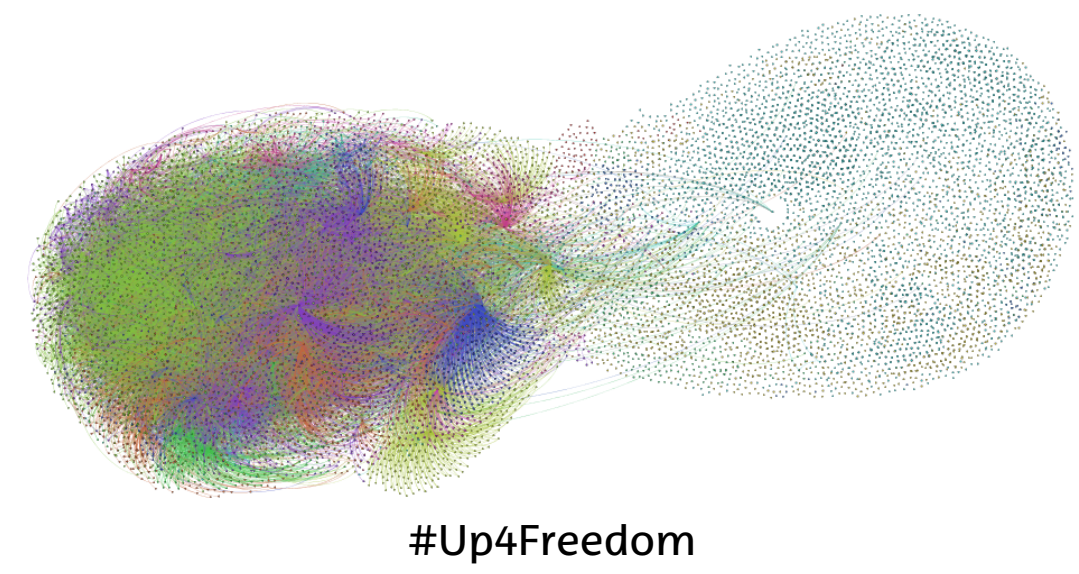

Fuente: Diseño basado en los datos obtenidos y refinados

Los siguientes grafos de red (figura 7) muestran algunas de las comunidades halladas en el procedimiento de manera particular. El algoritmo de detección de comunidades ha dado lugar a cinco comunidades que representan menos del $0,1 \%$ de los nodos de la red. Puesto que la prueba de Modularidad presenta graves limitaciones en la detección de comunidades pequeñas (Fortunato y Barthélemy, 2007), éstas no son consideradas en el análisis. Las siguientes quince son las comunidades que representan a más del $0,1 \%$ de los nodos de la red.

Figura 7. Comunidades detectadas en el grafo de red \#BasquesDecide y \#Up4Freedom separadas con representación superior al $0.1 \%$

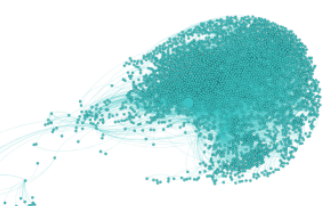

Comunidad 0

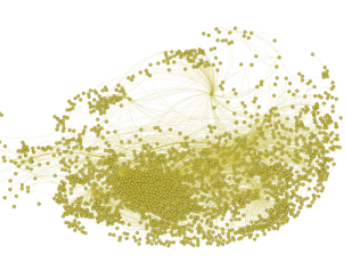

Comunidad 5

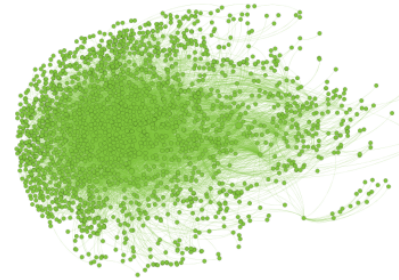

Comunidad 9 


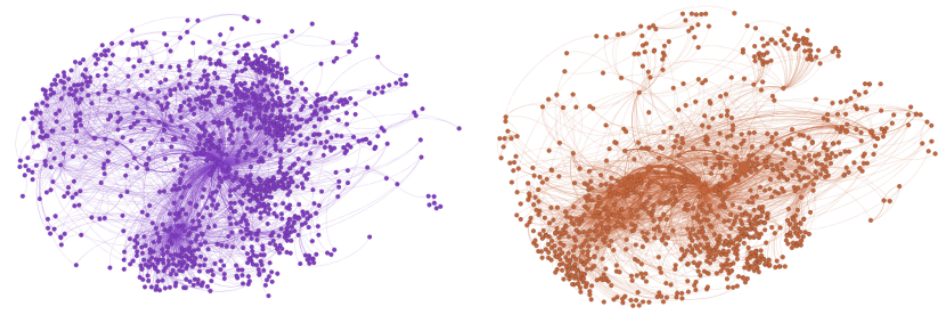

Comunidad 11

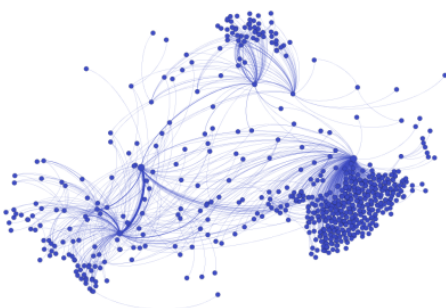

Comunidad 10

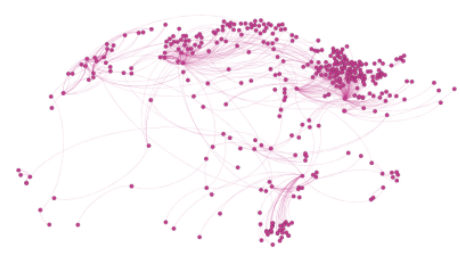

Comunidad 12
Comunidad 6

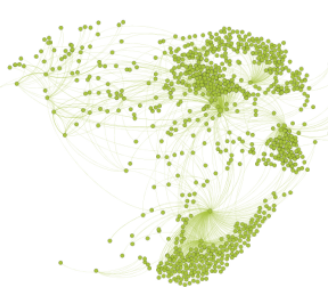

Comunidad 13

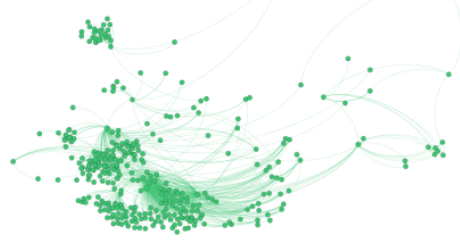

Comunidad 3

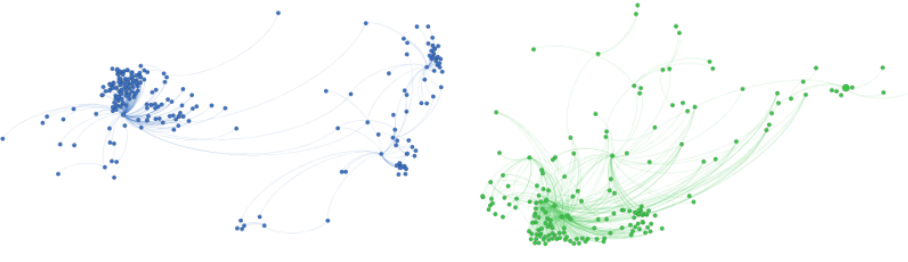

Comunidad 4

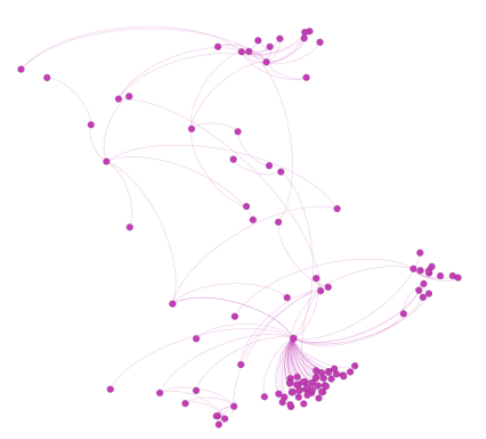

Comunidad 18
Comunidad 2

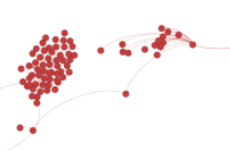

Comunidad 8

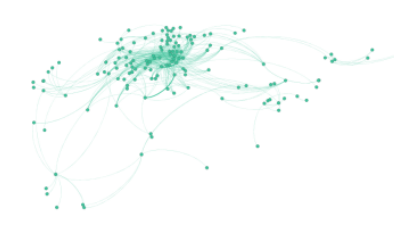

Comunidad 17

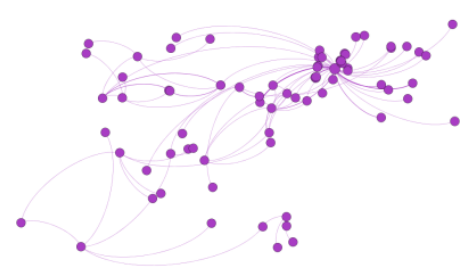

Comunidad 1

Fuente: Diseño basado en los datos obtenidos y refinados 
Entre las comunidades que contienen más del $5 \%$ de los nodos, las comunidades 0 y 5 son las que contienen fundamentalmente enlaces relativos a la etiqueta \#BasquesDecide, mientras que las comunidades 9 , 11, 6 y 13 los tienen de \#Up4Freedom.

La comunidad 0 destaca por la Centralidad de Intermediación de nodos como @gureeskudago, @argia, @berria o @eitbnoticias. La comunidad 5 por nodos como@naiz_info, @ahotsainfo, @garanet o @info7irratia. Se trata de comunidades reunidas en torno a proyectos colectivos con un grado elevado de estabilidad y consolidación, salvo por la recientemente creada organización convocante. En menor medida, la importancia de personajes públicos del mundo político y cultural es también notoria.

En la comunidad 9 destacan por su Centralidad de Intermediación usuarios propios de grupos locales o sectoriales de la ANC (e.g. @taxistesindepes, @ancgranollers, @blanesxindep) o de colles castelleres (e.g. @borinots, @verds). Sin embargo, siete de los diez usuarios más centrales en intermediación son individuos. La comunidad 11 es en la que más colectivos aparecen como centrales en intermediación entre los diez primeros nodos (i.e. @omnium, @catalanassembly, @anc_ireland y @assemblea); el resto son también usuarios individuales.

Entre los diez usuarios con mayor Centralidad de Intermediación de las comunidades 6 y 13 prácticamente solo hay individuos. A modo de curiosidad, cabe destacar que en la comunidad 13 se ubica una personalidad importante de la izquierda independentista vasca, aunque se trate de una comunidad fundamentalmente enlazada por la etiqueta \#Up4Freedom.

Todas estas comunidades agrupan gran parte de los nodos que en la consideración por separado de \#BasquesDecide y \#Up4Freedom conformaban comunidades distintas. Sin embargo, la agrupación en macrocomunidades de los nodos presentes en el conjunto de datos extraído de la convocatoria de Gure Esku Dago tiene como consecuencia la disolución en comunidades mayores de aquellas comunidades presididas por usuarios individuales, mientras que esto no pasa en el caso de \#Up4Freedom. 


\section{DISCUSIÓN}

Las diferencias entre las redes generadas en base a las etiquetas \#BasquesDecide y \#Up4Freedom son sustanciales. En primer lugar, el análisis muestra que los niveles de centralización de ambas redes son distintos, estando \#BasquesDecide bastante más centralizada que \#Up4Freedom tanto por lo que respecta al grado de entrada como al de salida. Esto se explica por la mayor participación de los nodos en la segunda instancia comunicativa. Una explicación razonable que justificaría las diferencias de participación seria el distinto grado de consolidación del paradigma de la auto-comunicación masiva en los dos territorios. Cabe entender también que los momentos sociales y políticos en el País Vasco y en Cataluña son significativamente distintos. En Cataluña el debate soberanista está a la orden del día, especialmente en las redes sociales virtuales, y en el País Vasco el mismo debate no goza de tanta popularidad en el momento actual.

Otra diferencia significativa consiste en el tipo de nodos que sostienen mayores cifras de Centralidad de Intermediación. Recordemos que la Centralidad de Intermediación viene determinada por la posición geodésica de un nodo en la red, otorgando mayor poder, influencia o referencialidad a aquellos usuarios que presiden en un cuello de botella comunicativo (Tsvetovat y Kouznetsov, 2011: 51). Mientras que el peso de los medios de comunicación es muy relevante en el caso de \#BasquesDecide, no lo es tanto para \#Up4Freedom. Una explicación posible para ello sería una mayor consolidación de las comunidades virtuales independentistas en el caso catalán, derivada de la centralidad del debate soberanista en sí. Por el contrario, en el caso vasco gran parte de la comunicación en redes sociales como Twitter estaría mediada por actores más tradicionales en los procesos comunicativos. Una lectura complementaria a la anterior sería la mayor consolidación de los a veces llamados "medios propios" del movimiento independentista vasco en comparación con el catalán.

La consideración conjunta de las comunidades en ambas redes tiene como consecuencia una mayor reducción de la complejidad social en la red construida bajo la etiqueta \#BasquesDecide. Sirva como ejemplo la imputación de los usuarios más centrales en intermediación de la comunidad 3 de la red original de \#BasquesDecide -la primera en importancia cuya centralidad reposa principalmente en usuarios individuales - en la comunidad 0 de la red combinada, presidida por la 
organización convocante y medios de comunicación. De hecho, las comunidades 0 y 5 agrupan el 46,35\% de los nodos de la red conjunta, mientras que en el caso de los enlaces establecidos en base a \#Up4Freedom es necesaria la consideración de las ocho comunidades restantes que agrupan a más del $1 \%$ de los nodos para lograr una cifra parecida de representación. Una lectura de tales resultados bajo las claves expuestas anteriormente, considerando que las comunidades en una red son lazos narrativos inestables que albergan la potencialidad para crear identidades colectivas suscita la idea de una mayor heterogeneidad narrativa en la arena comunicativa catalana. Habría entonces agentes más diversos contribuyendo a una narración de los hechos en el debate bajo la etiqueta \#Up4Freedom, configurada por menos usuarios pero más participativos que los de \#BasquesDecide.

Es conveniente considerar también algunas explicaciones alternativas a todos los fenómenos expuestos que pondrían en cuarentena el análisis efectuado en base a los resultados obtenidos. Se podría argumentar que los hechos sociales invocados en ambas etiquetas son distintos $y$, en buena medida, incomparables - las diferencias observadas en términos de Centralidad de Grado, entre el tipo de usuarios que gozan de altos niveles de Centralidad de Intermediación o entre las mismas dinámicas comunicativas que configuran comunidades quedarian neutralizadas-. Esto es parcialmente cierto, puesto que la convocatoria de la cadena humana tuvo una importancia contextual mayor ante la cual algunos medios de comunicación probablemente acudieron con más fuerza, mientras que en el caso catalán hay que entender la convocatoria simplemente como "una más" entre tantas hechas recientemente para reivindicar el derecho a decidir. Sin embargo, no son pocos los puntos de conexión entre ambas etiquetas: se trata de reivindicaciones homólogas, hechas durante el mismo periodo temporal, enfocadas hacia un público internacional aunque su participación es esencialmente vasca y catalana, y además, que se entienden como mutuamente referenciales tal y como se puede observar en la figura 6 , que refleja una penetración mutua y patrones comunicativos entre nodos presentes en ambos conjuntos de datos.

Quedan muchos interrogantes abiertos. Por ejemplo: ¿podemos hablar de patrones de desarrollo distintos del paradigma de la auto-comunicación masiva en el País Vasco y en Cataluña? De ser así, cabría identificar por un lado las causas de tal desarrollo dispar y por el otro las consecuencias del 
mismo. Mi hipótesis es que, en clave identitaria, si se sitúa el paradigma comunicativo emergente en el explanandum hallaremos principalmente diferencias coyunturales más que estructurales. Es decir, serán las propias relaciones intergrupales entre individuos auto-categorizados los que estarán dando lugar a las diferencias de consolidación del modelo comunicativo emergente más que el contenido en sí de las cargas identitarias disponibles. Ahora bien, si situamos el paradigma entrante de la auto-comunicación masiva del lado del explanans, cabría articular estrategias propiamente narrativas para comprender el tipo de lazos dispares que estarían emergiendo de sendas interacciones sociales.

\section{CONCLUSIONES}

Este artículo toma como punto de partida el paradigma de la autocomunicación de Manuel Castells (2000, 2009), situado en los procesos transformativos propios de la Sociedad-Red emergente. Éste constituye un modelo comunicativo que destaca por su horizontalidad, su demanda de participación y por la individualización de los protocolos de acceso a, y distribución de la información. Tal y como señala la colaboradora habitual de Castells, Imma Tubella (2004), se trata de transformaciones paradigmáticas que alteran el proceso occidental de construcción identitaria, dificultando la implementación de procesos de construcción de identidad "desde arriba" y multiplicando los narradores y las narraciones sobre los grupos en base a los que los individuos se vinculan.

Se ha considerado también la interpretación de las comunidades matemáticamente extraídas de una red mediante herramientas de Análisis de Redes Sociales como lazos narrativos que albergan la capacidad de devenir categorias identitarias operativas. Para ello ha sido esbozado un concepto "enredado" de identidad basado en perspectivas tan diferentes entre ellas como el paradigma psicosociológico de la Identidad Social, la teoría del Actor-Red de Bruno Latour o la concepción narrativa de las identidades colectivas del sociólogo Klaus Eder.

Posteriormente se han presentado las herramientas necesarias para abordar el análisis empírico de los conjuntos de datos construidos en base a las etiquetas de Twitter \#BasquesDecide y \#Up4Freedom y se ha procedido a la exposición y la interpretación de sus resultados. Se trata de una interpretación rescindida a los resultados obtenidos, dada la ya comentada escasez de artículos con propósitos análogos. Por un lado, se 
observan diferencias por lo que respecta la Centralidad de Grado entre ambas redes tanto en términos de grado de entrada como de salida. Tal efecto es consecuencia directa de la participación dispar de los usuarios que componen las redes, cosa que podría ser leída en términos de consolidación del paradigma comunicativo emergente, además de por un conjunto de explicaciones adicionales como los momentos políticos y sociales diferentes que atraviesan el País Vasco y Cataluña, o como la misma naturaleza de las etiquetas \#BasquesDecide y \#Up4Freedom y los temas que en ellas se tratan. Sea como fuere, los resultados abren la puerta a nuevos interrogantes e hipótesis que deben ser abordados mediante más instancias comunicativas y con métodos de investigación también diversos.

Por otro lado, se observan igualmente diferencias importantes en el tipo de nodos que gozan de mayor poder comunicativo en la red y que son capaces de interconectar a más individuos. Mientras que los datos referentes al caso catalán sugieren altos niveles de individualización del poder o la influencia en la red, en el caso vasco son organizaciones y, sobre todo, medios de comunicación privados de corte vascoparlante y/o soberanista los que estarían ejerciendo el mismo rol. Una vez más, los resultados sugieren grados de consolidación diversos de las comunidades identitarias soberanistas vasca y catalana en Twitter, así como grados de heterogeneidad dispares y estrategias comunicativas distintas en el seno de las mismas. Todo ello no puede considerarse sin tener en cuenta la especial importancia que algunos medios de comunicación vascos otorgaron a la cadena humana organizada por Gure Esku Dago y a la etiqueta \#BasquesDecide.

Estas estrategias distintas -que en el caso vasco se muestra más dependiente de los medios de comunicación de corte tradicional- y grados de heterogeneidad diferentes se pueden observar también en la partición resultante de los algoritmos de identificación de comunidades. Las comunicaciones establecidas bajo la etiqueta \#Up4Freedom dan lugar a más comunidades, lógicamente más pequeñas, cuyos nodos establecen relaciones internas significativamente más densas que con el resto. En ambos casos, el tipo de agente que contribuye con más fuerza a la unidad de la red son actores individuales - se trata de comunicaciones en una plataforma que propicia precisamente estas dinámicas-. A pesar de ello, como ya se ha comentado, la relevancia de agentes diferentes a individuos 
particulares es distinta en ambas redes, también en términos de las comunidades que las habitan.

Sería un error vincular las comunidades halladas con un concepto fuerte de identidad colectiva. Es la naturaleza inestable de una estructura social enredada la que da lugar a los grupos encontrados, y en ningún caso deben considerarse, al menos provisionalmente, categorías identitarias con capacidades de orientar significativamente la acción social de los individuos que se identifican con ellas. También sería inadecuado extrapolar las conclusiones aquí presentadas al conjunto de los procesos identitarios soberanistas vascos y catalanes que tienen lugar en Twitter, y que decir, a la realidad social offline. Sin embargo, entiendo que la investigación desarrollada sí que proporciona ideas altamente sugerentes para el abordaje de entornos distintos a los aquí tratados. El análisis comparativo de más casos particulares y un acercamiento propiamente narrativo a las comunidades encontradas serían una muy buena forma de profundizar en la comprensión de los procesos de configuración de las identidades políticas soberanistas contemporáneas en el País Vasco y en Cataluña.

\section{BIBLIOGRAFÍA}

Abrams, D., M.A. Hogg, 2001, "Collective Identity: Group Membership and Self-Conception", en M.A. Hogg y R.S. Tindale (Eds.), Blackwell Handbook of Social Psychology: Group Processes, Blackwell Publishers Ltd, Oxford-England, pp. 425-460.

Anderson, B., 1991, Imagined Communities: Reflections on the Origin and Spread of Nationalism, Verso, London.

Blondel, V.D., J.L. Guillaume, R. Lambiotte, E.L.J.S. Mech, 2008, "Fast Unfolding of Communities in Large Networks.", en Journal of Statistical Mechanics: Theory and Experiment, vol. 2008, pp. 1000810020.

Borgatti, S.P., M.G. Everett, J.C. Johnson, 2013, Analyzing Social Networks, SAGE Publications, London.

Brandes, U., 2001, "A Faster Algorithm for Betweenness Centrality", en Journal of Mathematical Sociology, vol. XXV, pp. 163-177.

Castells, M., 2000, "Materials for an Exploratory Theory of the Network society", en The British Journal of Sociology, vol LI/1, pp. 5-24.

Castells, M., 2009, Communication Power, OUP, Oxford. 
Castells, M., 2010, “Communal Heavens: Identity and Meaning in the Network Society", en M. Castells (Ed.), The Power of Identity: The Information Age: Economy, Society, and Culture Volume II, WileyBlackwell, Chichester, pp. 5-70.

Eder, K., 2009, "A Theory of Collective Identity: Making Sense of the Debate on a "European Identity", en European Journal of Social Theory, vol. XII/4, pp. 427-447.

Fortunato, S., M. Barthélemy, 2007, "Resolution Limit in Community Detection", en Proceedings of the National Academy of Sciences, vol. CIV/1, pp. 36-41.

Freeman, L. C., 1978, "Centrality in Social Networks Conceptual Clarification.", en Social Networks, vol. I/3, pp. 215-239.

Gellner, E., 2006, Nations and Nationalism, Blackwell Pub, Oxford.

Gu, B., P. Konana, R. Raghunathan y H.M. Chen, 2014, "The Allure of Homophily in Social Media: Evidence from Investor Responses on Virtual Communities", en Information Systems Research, vol. XXV/3, pp. 604-617.

Gure Esku Dago, 2014, “Cadena humana Durengo - Iruñea”, consultable en: https://gureeskudago.eus/. Última visita: 13 de marzo de 2015.

Hands, J., 2011, @ Is For Activism: Dissent, Resistance And Rebellion In A Digital Culture, Pluto Press, London.

Hanneman, R.A., M., Riddle, 2005, Introduction to Social Network Methods, University of California, Riverside.

Hechter, M., M. Levi, 1979, "The Comparative Analysis of Ethnoregional Movements.", en Ethnic and Racial Studies, vol. II/3, pp. 260-274.

Hobsbawm, E., T. O. Ranger, 1992, The Invention of Tradition, CUP, Cambridge.

Jacomy, M., T. Venturini, S. Heymann, M. Bastian, 2014, “ForceAtlas2, a Continuous Graph Layout Algorithm for Handy Network Visualization Designed for the Gephi Software.", en PLoS ONE, vol. IX/6, e98679.

Java, A., X. Song, T. Finin, B. Tseng, 2007, “Why We Twitter: Understanding Microblogging Usage and Communities", en Procedings of the Joint 9th WEBKDD and 1st SNA-KDD Workshop 2007, Springer, pp. 56-65.

Kelley, P. G., M. Sleeper, J. Cranshaw, 2013, "Conducting Research on Twitter: A Call for Guidelines and Metrics", consultable en: http://patrickgagekelley.com/papers/twitter-pmj.pdf. Última visita: 13 de marzo de 2015. 
Lambiotte, R., J-c Delvenne, M. Barahona, 2009, “Laplacian Dynamics and Multiscale Modular Structure in Networks", en ArXiv, 0812.1770.

Latour, B., 2011, “Networks, Societies, Spheres: Reflections of an ActorNetwork Theorist", en International Journal of Communication, vol. V/0, pp. 796-810.

Latour, B., 2005, Reassembling the Social: An Introduction to Actor-NetworkTheory, OUP, Oxford.

Lazarsfeld, P. F., R. K. Merton, 1954, "Friendship as a Social Process: A Substantive and Methodological Analysis" en M. Berger, T. Abel, y C. $\mathrm{H}$. Page (Eds.), Freedom and Control in Modern Society, Van Nostrand, New York, pp. 18-66.

Lozares, C., 1996, “La teoria de redes sociales”, en Papers, n 48, pp. 103-126.

Maalouf, A., 2001, In the Name of Identity: Violence and the Need to Belong, Arcade Pub, New York.

Molina, J.L., 2001, El análisis de Redes Sociales: Una introducción, Edicions Bellaterra, Barcelona.

Monsalve Moreno, M., 2008, "Análisis de Redes Sociales: Un Tutorial", consultable en: http://homepage.divms.uiowa.edu/ mmonsalv/bio/SNA.pdf. Última visita: 13 de marzo de 2015.

Newman, M., 2004, "Analysis of Weighted Networks", en Physical Review E, vol. LXXX/5, pp. 056131-056140.

Òmnium Cultural, 2014, "Catalans Want to Vote. Human Towers for Democracy", consultable en: https://www.omnium.cat/. Última visita: 13 de marzo de 2015.

Osborne, N., 2012, “Continuing Professional Development in Collaborative Social Media Spaces", consultable https://sites.google.com/site/cpdaandsocialmedia/. Última visita: 13 de marzo de 2015.

Requena Santos, F., 1989, “El concepto de Red Social”, en Revista Española de Investigaciones Sociológicas, $\mathrm{n}^{\circ}$ 48, pp. 48-89.

Rodríguez Díaz, J.A., 2005, "Análisis estructural y de redes”, en Cuadernos Medodológicos del CIS, vol XVI.

Seifi, M., I. Junier, J.B. Rouquier, S. Iskrov, J.L Guillaume, 2013, “Stable Community Cores in Complex Networks.", en R. Menezes, A. Evsukoff y M. González (Eds.), Complex Networks, Studies in Computational Intelligence, Springer, New York, pp. 87-98. 
Stassen, W., 2011, "Your News in 140 Characters: Exploring the Role of Social Media in Journalism", en Global Media Journal African Edition, vol. IV/1, pp. 116-131.

Tajfel, H., 1974, "Social Identity and Intergroup Behaviour.", en Social Science Information, vol. XIII/2, pp. 65-93.

Tajfel, H., J. C. Turner, 1979, "An Integrative Theory of Intergroup Conflict.", en W. G. Austin y S. Worchel (Eds.), Psychology of Intergroup Relations, Brooks/Cole, Monterey, pp. 33-47.

Tsvetovat, M., A. Kouznetsov, 2011, Social Network Analysis for Startups: Finding Connections on the Social Web, Reilly Media.

Tubella, I., 2004, "Television, the Internet, and the Construction of Identity", en M. Castells (Ed.), The Network Society A Cross-Cultural Perspective, Edward Elgar Publishing, Inc., Cheltenham, pp. 257-268.

Turner, J.C., 1999, "Some Current Issues in Research on Social Identity and Self Categorization Theories", en N. Ellmers y R. Spears (Eds.), Social identity: Context, Commitment, Content, Blackwell Pub., London pp. 634.

Turner, J.C., P.J. Oakes, S.A. Haslam, C. McGarty, 1994, "Self and Collective: Cognition and Social Context", en Personality and Social Psychology Bulletin, vol. XX/5, pp. 454-463.

Twitter, 2015, "About Twitter", consultable en: https://about.twitter.com/company. Última visita: 13 de marzo de 2015.

Wasserman, S., K. Faust, 1994, Social Network Analysis: Methods and Applications. CUP, Cambridge. 